\title{
Gestational Diabetes Mellitus and Infant Adiposity at Birth: A Systematic Review and Meta-Analysis of Therapeutic Interventions
}

\author{
Manoja P. Herath (D), Jeffrey M. Beckett (D), Andrew P. Hills, Nuala M. Byrne and Kiran D. K. Ahuja * \\ School of Health Sciences, College of Health and Medicine, University of Tasmania, Launceston, \\ TAS 7248, Australia; manoja.herath@utas.edu.au (M.P.H.); jeffrey.beckett@utas.edu.au (J.M.B.); \\ andrew.hills@utas.edu.au (A.P.H.); nuala.byrne@utas.edu.au (N.M.B.) \\ * Correspondence: kiran.ahuja@utas.edu.au
}

\section{check for} updates

Citation: Herath, M.P.; Beckett, J.M.; Hills, A.P.; Byrne, N.M.; Ahuja, K.D.K Gestational Diabetes Mellitus and Infant Adiposity at Birth: A

Systematic Review and

Meta-Analysis of Therapeutic Interventions. J. Clin. Med. 2021, 10 835. https://doi.org/10.3390/jcm 10040835

Academic Editor:

Charikleia Stefanaki

Received: 6 January 2021

Accepted: 16 February 2021

Published: 18 February 2021

Publisher's Note: MDPI stays neutral with regard to jurisdictional claims in published maps and institutional affiliations.

Copyright: (c) 2021 by the authors. Licensee MDPI, Basel, Switzerland. This article is an open access article distributed under the terms and conditions of the Creative Commons Attribution (CC BY) license (https:// creativecommons.org/licenses/by/ $4.0 /)$.
Abstract: Exposure to untreated gestational diabetes mellitus (GDM) in utero increases the risk of obesity and type 2 diabetes in adulthood, and increased adiposity in GDM-exposed infants is suggested as a plausible mediator of this increased risk of later-life metabolic disorders. Evidence is equivocal regarding the impact of good glycaemic control in GDM mothers on infant adiposity at birth. We systematically reviewed studies reporting fat mass (FM), percent fat mass (\%FM) and skinfold thicknesses (SFT) at birth in infants of mothers with GDM controlled with therapeutic interventions (IGDMtr). While treating GDM lowered FM in newborns compared to no treatment, there was no difference in FM and SFT according to the type of treatment (insulin, metformin, glyburide). IGDMtr had higher overall adiposity (mean difference, 95\% confidence interval) measured with FM (68.46 g, 29.91 to 107.01$)$ and $\% \mathrm{FM}(1.98 \%, 0.54$ to 3.42$)$ but similar subcutaneous adiposity measured with SFT, compared to infants exposed to normal glucose tolerance (INGT). This suggests that IGDMtr may be characterised by excess fat accrual in internal adipose tissue. Given that intra-abdominal adiposity is a major risk factor for metabolic disorders, future studies should distinguish adipose tissue distribution of IGDMtr and INGT.

Keywords: gestational diabetes mellitus; treatment; adiposity; fat mass; skinfold thickness; newborns; infants

\section{Introduction}

The prevalence of gestational diabetes mellitus (GDM) is rising globally, affecting up to $38 \%$ of pregnancies in some populations [1]. As well as causing complications during pregnancy and delivery including macrosomia, shoulder dystocia and preterm birth, exposure to GDM in utero places offspring at an increased risk of obesity and type 2 diabetes in later life [2,3]. The mechanisms associated with this increased risk of obesity and type 2 diabetes are not well understood; however, increased adiposity during foetal growth has been suggested as a potential mediator [4]. The Pedersen hypothesis [5] suggests that, as glucose freely crosses the placenta, maternal hyperglycaemia in diabetic pregnancies leads to foetal hyperinsulinaemia, causing accelerated foetal uptake of glucose (foetal glucose steal phenomenon) and deposition of excess foetal adipose tissue [6]. The impact of GDM on adipose tissue growth in the foetus can be identified with adiposity measures at birth, for example, fat mass (FM), percent fat mass (\%FM) and skinfold thickness (SFT) [7].

Diagnosis and management of GDM continue to be controversial. The earlier definition of GDM, i.e., "any degree of glucose intolerance that occurs or is first diagnosed during pregnancy" [8], was used for many years and enabled a uniform approach to the detection of GDM. However, the classification of women with unrecognized overt diabetes as GDM and providing treatments accordingly may not be effective because risks associated with type 1 and type 2 diabetes are greater than GDM [9]. In the latest clinical 
practice recommendations by the American Diabetes Association [10], GDM is defined as "glucose intolerance first diagnosed during the second or third trimester of pregnancy in women without overt diabetes prior to pregnancy, which resolves postnatally", and this involves risk-based screening for type 2 diabetes or prediabetes at their initial prenatal visit. Nonetheless, different criteria are currently being used worldwide to diagnose GDM. A landmark change in these diagnostic thresholds occurred when the Hyperglycaemia and Adverse Pregnancy Outcome (HAPO) study [11] demonstrated a positive linear association between increasing levels of plasma glucose and adverse pregnancy outcomes and subsequently, lowered thresholds for screening GDM. These new diagnostic thresholds (fasting plasma glucose 5.1-6.9 mmol/L, 1-h plasma glucose $\geq 10.0 \mathrm{mmol} / \mathrm{L}$ or $2-\mathrm{h}$ $8.5-11.0 \mathrm{mmol} / \mathrm{L}$ ) were promulgated by the International Association of Diabetes and Pregnancy Study Groups (IADPSG) in 2010 and by the World Health Organisation (WHO) in 2013, and this enabled detection of more GDM cases [12].

Awareness of the adverse outcomes associated with GDM has been a driver for substantial improvements in perinatal care for pregnant women with GDM in recent years [13]. The first-line treatment for GDM involves lifestyle changes, e.g., modified diet and increased physical activity, and nearly two-thirds of women can achieve glycaemic targets with this approach [14]. When blood glucose levels are not adequately controlled with modified lifestyle alone, supplementary pharmacological treatments such as metformin, glyburide or insulin are added to the therapeutic regimen [15]. Glycaemic control in GDM women using modified dietary interventions alone has resulted in lower birth weights and less macrosomia [16], despite the high heterogeneity in diet observed among different populations [17]. Similarly, using pharmaceutical interventions along with or without lifestyle changes has resulted in reduced risk of macrosomia [18] and has prevented GDMassociated adverse health conditions in neonates [19]. Nevertheless, the effect of GDM treatments on neonatal adiposity is understudied, and the evidence for whether good glycaemic control in GDM can normalise foetal adiposity is contradictory [20-22]. To ascertain the impact of glycaemic control in GDM on infant adiposity at birth, we systematically reviewed studies reporting adiposity in newborns of mothers with GDM controlled with therapeutic interventions.

\section{Materials and Methods}

This work was conducted following the Preferred Reporting Items for Systematic Reviews and Meta-Analyses (PRISMA) guidelines [23]. Our protocol is registered in PROSPERO (CRD42020175338).

\subsection{Search Strategy}

Electronic searches were conducted in three stages with the assistance of a Research Librarian at the University of Tasmania, Australia. First, a limited search was undertaken in Medline and Scopus, using search terms: "gestational diabetes", "body composition" and "infants". The title, abstract and index terms of the retrieved articles were scanned to build a keyword list. In the second step, a broader search was conducted (March 2020), using the identified terms in MEDLINE in Ovid, Embase, CINHAL, PubMed and Web of Science databases, limiting the results to studies published in "English" language, "human" species and "infants" age group. The search strategy for MEDLINE is shown in Figure S1, and a similar approach was used in other databases. Finally, we manually scanned the reference lists of included articles, relevant reviews, and citations to identify any additional studies. Hand searches were not conducted for any specific journal, and we did not trace any grey literature.

\subsection{Eligibility Criteria}

We included all study types reporting adiposity in infants exposed to GDM. Inclusion criteria were: (1) data collected at birth or $<1$-month infants' age; (2) availability of infant adiposity measure(s); i.e., fat mass (FM), percent fat mass (\%FM) or skinfold thickness (SFT); 
and (3) availability of information regarding what therapeutic measures were undertaken to control GDM. Exclusion criteria were (1) examination of maternal glycaemia as a continuous variable; (2) assessment of only foetal measurements (e.g., ultrasound scans); (3) merging of data for GDM exposed infants with pregestational diabetes-exposed infants; (4) full report of the study not published in English; and (5) review articles, protocol papers and conference abstracts. When there were multiple publications from the same sample of study participants, we only included the paper that presented the most appropriate data for the purpose of this review.

\subsection{Study Selection}

The results emanating from database searches were imported into the Covidence software ${ }^{\circledR}$ [24]. After removing duplicates, the search outputs were independently reviewed at the title and abstract level by M.P.H. and K.D.K.A./J.M.B. to find potentially eligible articles. These articles were screened at the full-text level by the same reviewers to determine the eligibility of the papers for data extraction.

\subsection{Quality Assessment}

The methodological quality of the selected studies was assessed by two reviewers (M.P.H. and J.M.B) using the Evidence Project risk of bias tool. This tool is appropriate for assessing study rigour for both randomised and non-randomised intervention studies. The Evidence Project risk of bias tool includes eight items: (1) cohort, (2) control or comparison group, (3) pre-post intervention data, (4) random assignment of participants to the intervention, (5) random selection of participants for assessment, (6) follow-up rate of $80 \%$ or more, (7) comparison groups equivalent on sociodemographics, and (8) comparison groups equivalent at baseline on outcome measures [25]. For criterion 7, we considered infant sex and ethnicity as the relevant sociodemographic characteristics. If authors reported that study arms were equivalent on only one sociodemographic variable, we considered the meeting of the criterion as "Partial". Additionally, if the study arms were not equivalent on at least one sociodemographic variable, we considered that the criterion was not met. Any disagreements between the two reviewers regarding the inclusion of studies and quality assessment were resolved by discussion and consensus.

\subsection{Data Extraction}

A pre-designed data collection form was used to extract information from each paper. This information included: (1) study characteristics (author, year, design, time period of data collection, state/country of the study, inclusion and exclusion criteria), (2) study groups (sample size, male\%), (3) method(s) (GDM screening/diagnostic criteria, treatments to control GDM, target blood glucose level, degree of glycaemic control, body composition measurement technique) and (4) outcomes (FM, \%FM, SFT).

\subsection{Data Analysis}

For the purpose of data synthesis, the included studies were categorised according to comparison groups: (1) 'treated' GDM vs. 'untreated' GDM; (2) different treatment regimens for GDM and, (3) treated GDM vs. normal glucose tolerance (NGT). When blood glucose levels of GDM mothers were controlled with any form of therapeutic intervention (including lifestyle modification and/or pharmaceutical interventions), they were considered as 'treated', and usual antenatal care without any specific treatment for GDM was considered as 'untreated'. When an adequate number of studies were available, meta-analyses were performed with the inverse variance statistical method and random effects analysis model (RevMan version: 5.4.0) [26]. Mean difference at a 95\% confidence interval was used to combine the results. Forest plots were used to demonstrate the outcomes. Heterogeneity between the studies in meta-analyses was determined with a $\mathrm{Chi}^{2}$ test on the Q statistic (variance of the observed effect sizes in the meta-analysis), $\mathrm{Tau}^{2}$ (between-study variance of the true effect sizes) and $\mathrm{I}^{2}$ (proportion of the observed 
variation in the effect size due to differences in the true underlying effect sizes, as opposed to sampling error). An alpha level $<0.05$ was considered statistically significant. Potential sources of heterogeneity, i.e., level of glycaemic control in GDM mothers, any advances in the effectiveness of treatments for GDM in 'recent' years (defined as study data collection occurred during or after 2010: referred as post-2010) compared to 'pre-2010' (defined as study data collection occurred before 2010), GDM diagnosis criteria and body composition assessment technique, were investigated with subgroup analyses. Sensitivity testing was performed with 'leave-one-out' testing.

\section{Results}

\subsection{Study Selection}

Of the 1072 references identified through database searching, 19 matched inclusionexclusion criteria (Figure 1). An additional six papers were identified through a review of reference lists, relevant reviews, and forward citations. In total, 25 studies [7,20-22,27-47] were included in the systematic review, of which 17 [7,20-22,27-29,31,33,37-39,41-45] were included in the meta-analysis.

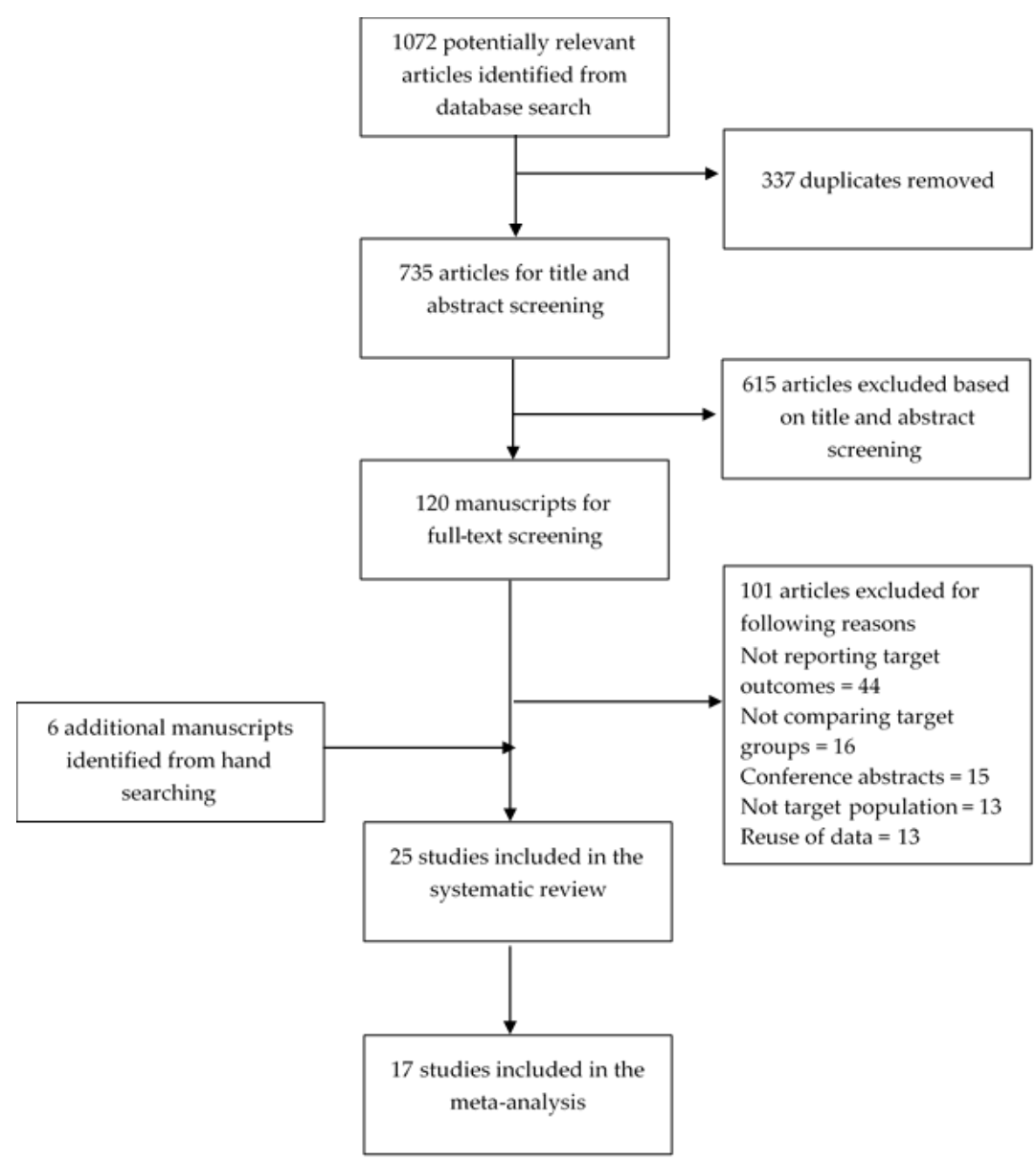

Figure 1. Flow of information through the different phases of the review.

\subsection{Description of the Studies}

The selected references included three randomised clinical trials [34-36], 15 cohort studies [7,21,27,29-31,37,38,41-47], 3 case-control studies [20,33,39,42] and 4 cross-sectional studies $[22,28,32,40]$. The included studies were published between 1980 and 2020, and from 12 different countries, i.e., the United States [7,28,29,31,35,36,40], Australia [21,22,45], New Zealand [30,33], Australia and New Zealand [34], Germany [37,38,41], Sweden [20,44], China [32], France [43], Italy [27], Malaysia [47], Spain [39], the United Kingdom [46] and Turkey [42]. Sample sizes varied from 25 to 1000 (Table 1). 
Table 1. Characteristics and findings of the studies.

\begin{tabular}{|c|c|c|c|c|c|c|c|}
\hline $\begin{array}{l}\text { First Author, Year, Study } \\
\text { Design, Time of Data } \\
\text { Collection, Location } \\
\end{array}$ & $\begin{array}{l}\text { Study Groups } n \\
\text { (Males\%) }\end{array}$ & GDM Identification/Definition & Treatment(s) & $\begin{array}{l}\text { Target Blood Glucose Levels (BGLs) } \\
\text { and Level of Glycaemic Control }\end{array}$ & Infants' Age & $\begin{array}{l}\text { Infants' Body Composition } \\
\text { Assessment } \\
\text { Method/SKINFOLD } \\
\text { Thickness Measurements }\end{array}$ & Findings \\
\hline $\begin{array}{c}\text { Landon, } 2009 \\
\text { Randomised trial } \\
\text { 2002-2007 } \\
\text { Bethesda, MD, USA [36] }\end{array}$ & $\begin{array}{l}\text { Control }=473 \\
\text { Treatment }=485\end{array}$ & $\begin{array}{l}\text { At } 24 \text { th and } 30 \text { th weeks using } 4 \text { th } \\
\text { International workshop conference } \\
\text { criteria }\end{array}$ & $\begin{array}{r}\text { (1) Treate } \\
\begin{array}{l}\text { Diet therapy }(n=427) \\
\text { and insulin }(n=36)\end{array}\end{array}$ & $\begin{array}{l}\text { M vs. no treatment for GDM } \\
\text { Targeted for fasting glucose } \\
<5.3 \mathrm{mmol} / \mathrm{L} \text { or } \\
\text { 2-h post-prandial glucose, } \\
<6.7 \mathrm{mmol} / \mathrm{L} \\
\text { Good glycaemic control achieved } \\
\end{array}$ & Birth & $\begin{array}{l}\text { FM was calculated as } \\
\text { proposed by Catalano et al., } \\
\text { 1995. } \\
\text { Flank skinfold } \\
\text { (data not given) } \\
\end{array}$ & $\begin{array}{l}\text { FM: Lower in treatment group } \\
(427 \pm 198 \text { vs. } 464 \pm 222, p=0.003)\end{array}$ \\
\hline \multicolumn{8}{|c|}{ (2) Different treatment regimens for GDM } \\
\hline $\begin{array}{c}\text { Simmons, 1997 } \\
\text { 1991-1992 } \\
\text { Middlemore Hospital and } \\
\text { National Women's Hospital, } \\
\text { Auckland, New Zealand [30] } \\
\end{array}$ & $\begin{array}{c}\text { All GDM } \\
\text { Non-insulin }=11 \\
\text { Insulin }=9(33 \%)\end{array}$ & $\begin{array}{l}\text { At } 28-32 \text { weeks } \\
\text { gestation, using } \\
\text { modified O'Sullivan } \\
\text { criteria }\end{array}$ & $\begin{array}{c}\text { All women } \\
\text { received } \\
\text { dietary therapy }\end{array}$ & $\begin{array}{l}\text { Targeted fasting glucose }>5.5 \\
\mathrm{mmol} / \mathrm{L} \text { and } / \text { or } 2-\mathrm{h} \text { post-prandial } \\
\text { glucose }>7.0 \mathrm{mmml} / \mathrm{L}\end{array}$ & $<24 \mathrm{~h}$ & Subscapular & $\begin{array}{c}\text { SFT: Not significantly different subscapular } \\
5.4(4.8-7.0) \text { vs. } 6.8(5.0-7.9)\end{array}$ \\
\hline $\begin{array}{c}\text { Rowan, 2008 } \\
\text { randomised, open-label trial } \\
\text { 10 New Zealand and } \\
\text { Australian urban } \\
\text { obstetrical hospitals [34] } \\
\end{array}$ & $\begin{array}{c}\text { All GDM } \\
\text { Metformin }=363 \\
\text { Insulin }=370\end{array}$ & $\begin{array}{l}\text { According to the criteria of the } \\
\text { Australasian of } \\
\text { Diabetes in Pregnancy } \\
\text { Society (ADIPS) }\end{array}$ & $\begin{array}{l}\text { Metformin }=363 \\
\text { Insulin }=370\end{array}$ & $\begin{array}{c}\text { Aimed for the capillary glucose } \\
\text { levels recommended by the ADIPS } \\
\text { (after an overnight fast, }<5.5 \\
\text { mmol } / \mathrm{L} ; 2 \text { 2h post-prandial level, } \\
<7.0 \mathrm{mmol} / \mathrm{L}\end{array}$ & $<48 \mathrm{~h}$ & Triceps and subscapular & $\begin{array}{c}\text { SFT: Metformin group not significantly different from } \\
\text { insulin group } \\
\text { triceps }(5.2 \pm 1.6 \text { vs. } 5.1 \pm 1.2, p=0.30) \\
\text { subscapular }(5.2 \pm 1.5 \text { vs. } 5.2 \pm 1.3, p=0.60)\end{array}$ \\
\hline \multicolumn{8}{|c|}{ (b) Studies that measured body composition } \\
\hline $\begin{array}{c}\text { Catalano, } 2003 \\
\text { Prospective cohort } \\
\text { 1990-2000 } \\
\text { Pregnancy Diabetes Clinic in } \\
\text { Cheveland } \\
\text { Ohio, USA [7] }\end{array}$ & $\begin{array}{l}\mathrm{NGT}=220(54 \%) \\
\mathrm{GDM}=195(51 \%)\end{array}$ & $\begin{array}{l}\text { National Diabetes Data Group } \\
\text { criteria }\end{array}$ & $\begin{array}{c}\text { Diet only }=128 \\
\text { Diet }+ \text { insulin }=67\end{array}$ & $\begin{array}{c}\text { Targeted fasting } \\
\text { glucose }>5.5 \mathrm{mmol} / \mathrm{L} \text { and } / \mathrm{or} 2 \mathrm{2h} \\
\text { post-prandial glucoses }>6.7 \mathrm{mmol} / \mathrm{L} ; \\
\text { Women maintained glucose values } \\
\text { within the target range with diet and } \\
\text { exercise }(66 \%) \text {, plus insulin }(34 \%)\end{array}$ & $<72 \mathrm{~h}$ & TOBEC & $\begin{array}{c}\text { FM: Higher in diet }+ \text { insulin group }(492 \pm 215 \text { vs. } \\
\text { 407 } \pm 196, p=0.006) \\
\text { \%FM: Higher in diet }+ \text { insulin } \\
\text { group }(13.6 \pm 4.6 \text { vs. } 11.7 \pm 4.5, p=0.007)\end{array}$ \\
\hline \multicolumn{4}{|c|}{ (3) Treated GDM vs. NGT } & & & FIGDMtr compared to INGT & 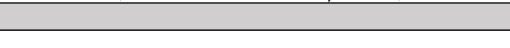 \\
\hline $\begin{array}{l}\text { Stevenson, } 1991 \\
\text { Crosss-sectional } \\
\text { USA [28] }\end{array}$ & $\begin{array}{l}\text { AGA NGT }=20 \\
\text { LGA NGT }=20 \\
\text { AGA GDM }=13\end{array}$ & $\begin{array}{l}\text { O'Sullivan and } \\
\text { Mahan criteria }\end{array}$ & Dietary control & 'Well-managed GDM' & $<72 \mathrm{~h}$ & Triceps & $\begin{array}{c}\text { Not significantly different } \\
\text { triceps compared to AGA NGT group (5.0 } \pm 1.1 \mathrm{vs} \text {. } \\
4.3 \pm 0.8, p>0.05) \text { and LGA NGT group }(5.0 \pm 1.1 \mathrm{vs} . \\
6.2 \pm 2.0, p=0.058)\end{array}$ \\
\hline
\end{tabular}


Table 1. Cont

\begin{tabular}{|c|c|c|c|c|c|c|c|}
\hline $\begin{array}{l}\text { First Author, Year, Study } \\
\text { Design, Time of Data } \\
\text { Collection, Location }\end{array}$ & $\begin{array}{l}\text { Study Groups } n \\
\text { (Males } \%)\end{array}$ & GDM Identification/Definition & Treatment(s) & $\begin{array}{l}\text { Target Blood Glucose Levels (BGLs) } \\
\text { and Level of Glycaemic Control }\end{array}$ & Infants' Age & $\begin{array}{l}\text { Infants' Body Composition } \\
\text { Assessment } \\
\text { Method/SKINFOL } \\
\text { Thickness Measurements }\end{array}$ & Findings \\
\hline $\begin{array}{c}\text { Vohr, } 1995 \\
\text { Prospective longitudinal } \\
\text { cohort } \\
\text { 1991-1993 } \\
\text { Women and Infants' hospital, } \\
\text { Rhode Island [29] }\end{array}$ & $\begin{array}{l}\text { AGA NGT }=69 \\
\text { AGA GDM }=62 \\
\text { LGA GDM }=57 \\
\text { LGA NGT }=74\end{array}$ & $\begin{array}{l}\text { Carpenter and } \\
\text { Coustan criteria }\end{array}$ & $\begin{array}{c}\text { Diet only }=385 \\
\text { Diet + insulin }=34 \\
\text { Diet includes } 45-50 \% \\
\text { carbohydrates, } 5 \% \text {. } 5 \% \\
\text { protein, and } 25 \% \text { fat. }\end{array}$ & $\begin{array}{l}\text { Targeted fasting glucose }>5.5 \\
\text { mmol/L and/or } 2 \text {-h post-prandial } \\
\text { glucose }>6.7 \mathrm{mmol} / \mathrm{L} \text {. The } \\
\text { management team worked with all } \\
\text { mothers to maintain BGL targets }\end{array}$ & $20 \pm 12 \mathrm{~h}$ & $\begin{array}{l}\text { Triceps, subscapular, } \\
\text { abdominal, suprailiac, and } \\
\text { medial calf SFT }\end{array}$ & 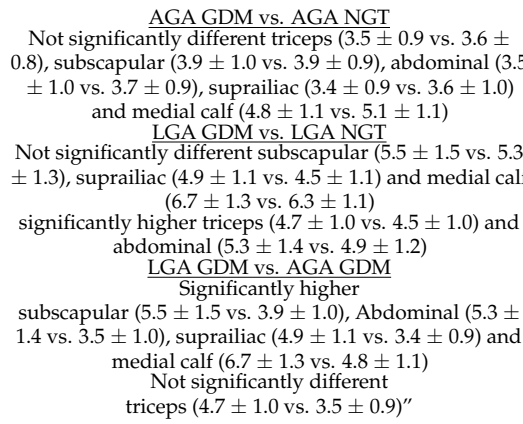 \\
\hline $\begin{array}{c}\mathrm{Ng}, 2004 \\
\text { Cross-sectional } \\
\text { Prince of Wales Hospital } \\
\text { Hong Kong [32] }\end{array}$ & $\begin{aligned} \mathrm{NGT} & =40(50 \%) \\
\mathrm{GDM} & =42(45.5 \%)\end{aligned}$ & ADIPS criteria (1998) & $\begin{array}{l}\text { Low-energy diet (1800 } \\
\mathrm{kcal} / \mathrm{d})\end{array}$ & Not reported & $<24 \mathrm{~h}$ & Triceps and subscapular & $\begin{array}{l}\text { SFT: } \\
\text { Not significantly different triceps }(4.8(4.2-5.1) \text { vs. } \\
4.7(4.1-5.5)) \text { and subscapular }(4.8(4.3-5.3) \text { vs. } \\
4.8(4.1-5.3), p>0.05)\end{array}$ \\
\hline $\begin{array}{l}\text { Westage, } 2006 \\
\text { case-control } \\
\text { 1999-2001 } \\
\text { Midlemore Hospital, } \\
\text { South Auckland } \\
\text { New Zealand [33] }\end{array}$ & $\begin{array}{c}\mathrm{NGT}=95 \\
\mathrm{GDM}=138\end{array}$ & $\begin{array}{c}\text { Local criteria for diagnosis of GDM } \\
\text { fasting } \\
\text { glucose } \geq 5.5 \mathrm{mmol} / \text { Land } / \text { or a } \\
\text { 2-h value atter a } 75 \mathrm{~g} \text { glucose load } \\
\geq 9.0 \mathrm{mmol} / 1\end{array}$ & $\begin{array}{l}\text { Insulin, usually as lispro } \\
\text { insulin up to } \\
\text { three times daily along with } \\
\text { Humulin N if target fasting } \\
\text { glucose exceeded two } \\
\text { occasions or } \\
\text { post-prandial } \\
\text { readings were } \\
\text { consistently high. }\end{array}$ & $\begin{array}{l}\text { Target fasting glucose }<5.5 \mathrm{mmol} / \mathrm{L} \\
\text { and post-prandial readings }<6.5 \\
\mathrm{mmol} / \mathrm{l} .\end{array}$ & $<24 \mathrm{~h}$ & Triceps and scapular & $\begin{array}{l}\text { SFT: } \\
\text { Significantly higher triceps }(5.0 \pm 1.2 \text { vs. } 4.4 \pm 1.0) \text { and } \\
\text { scapular }(5.6 \pm 1.6 \text { vs. } 4.4 \pm 1.0)\end{array}$ \\
\hline $\begin{array}{c}\text { Kara, 2017 } \\
\text { Cohort } \\
\text { Ataturk University, } \\
\text { Medical Hospital, } \\
\text { Erzurum, Turkey [42] }\end{array}$ & $\begin{array}{c}\mathrm{NGT}=20 \\
\mathrm{GDM}=15 \text { groups } \\
\text { were matched for } \\
\text { gestational age and } \\
\text { sex }\end{array}$ & $\begin{array}{l}\text { At 24-28 gestational week using } \\
\text { World Health Organisation (WHO) } \\
\text { criteria }\end{array}$ & $\begin{array}{l}\text { All were treated } \\
\text { with dietary intervention, } \\
\text { physical activity } \\
\text { recommendation, and } \\
\text { lifestyle management. All of } \\
\text { them (diabetic) have used } \\
\text { insulin therapy. }\end{array}$ & $\begin{array}{l}\text { While the mean HbA1c level of } \\
\text { mothers with gestational diabetes } \\
\text { was } 5.9 \pm 1.9 \% \text {, that of the controls } \\
\text { was } 5.2 \pm 0.33 \% \text {; there } \\
\text { was no significant } \\
\text { difference. Therefore, mothers with } \\
\text { gestational diabetes were well } \\
\text { controlled. }\end{array}$ & Birth & $\begin{array}{c}\text { Triceps, } \\
\text { Biceps, } \\
\text { subscapular }\end{array}$ & $\begin{array}{c}\text { SFT: } \\
\text { Significantly higher triceps }(3.9 \pm 0.7 \text { vs. } 3.3 \pm 1.1, p= \\
0.009) \text { and subscapular }(3.8 \pm 0.8 \mathrm{vs} .3 \pm 1.2 \pm 1.2, p=0.04) \\
\text { Not significantly different } \\
\text { biceps } \\
(2.8 \pm 0.6 \text { vs. } 2.6 \pm 0.9, p=0.32)\end{array}$ \\
\hline $\begin{array}{c}\text { Mitanchez, 2017 } \\
\text { prospective cohort } \\
\text { exposure-matched cohort } \\
\text { 2010-2013 } \\
\text { Paris, France [43] }\end{array}$ & $\begin{array}{l}\text { Lean NGT }=164 \\
\text { Lean GDM }=41 \\
\text { Obese NGT }=120 \\
\text { Obese GDM }=90\end{array}$ & 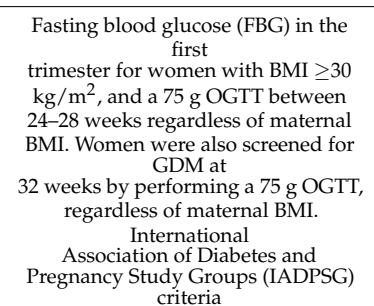 & $\begin{array}{l}\text { The first line treatment was } \\
\text { dietary intervention with a } \\
\text { standard } 1800 \text { kcal daily } \\
\text { meal plan } \\
\text { divided into three meals and } \\
\text { snacks. Insulin } \\
\text { treatment after two weeks of } \\
\text { failed dietary therapy. }\end{array}$ & $\begin{array}{l}\text { Target fasting glucose }<5.0 \mathrm{mmol} / \mathrm{L} \\
\text { and } \\
\text { post-prandial level }<6.7 \mathrm{mmol} / \mathrm{L} \text {. }\end{array}$ & $<72 \mathrm{~h}$ & $\begin{array}{l}\text { Triceps, biceps, suprailiac } \\
\text { and subscapular }\end{array}$ & $\begin{array}{c}\text { SFT: } \\
\text { Normal weight group } \\
\text { Not significantly different sum of SFF (triceps, biceps, } \\
\text { subscapular, suprailiac) } \\
(18.6 \pm 3.7 \text { vs. 17.8 } \pm 3.1, p>0.05) \\
\text { Obese group } \\
\text { Not significantly different sum of SFT } \\
(19.9 \pm 44 \text { vs. } 19.0 \pm 3.5, p>0.05)\end{array}$ \\
\hline
\end{tabular}


Table 1. Cont.

\begin{tabular}{|c|c|c|c|c|c|c|c|}
\hline $\begin{array}{l}\text { First Author, Year, Study } \\
\text { Design, , ime of Data } \\
\text { Collection, Location }\end{array}$ & $\begin{array}{l}\text { Study Groups } n \\
\text { (Males\%) }\end{array}$ & GDM Identification/Definition & Treatment(s) & $\begin{array}{l}\text { Target Blood Glucose Levels (BGLs) } \\
\text { and Level of Glycaemic Control }\end{array}$ & Infants' Age & $\begin{array}{l}\text { Infants' Body Composition } \\
\text { Assessment } \\
\text { Method/SKINFOLD } \\
\text { Thickness Measurements }\end{array}$ & Findings \\
\hline $\begin{array}{c}\text { Prentice, 2019 } \\
\text { Prospective cohort } \\
\text { 2001-2009 and 2011-2013 } \\
\text { Rosie Maternity Hospital, } \\
\text { Cambridge, UK [46] } \\
\text { (additional data provided by } \\
\text { authors) }\end{array}$ & $\begin{array}{c}\text { Earlier GDM }=98 \\
(53 \%) \\
\text { Recent GDM }=122 \\
(54 \%) \\
\text { Recent NGT }=876 \\
(52 \%)\end{array}$ & $\begin{array}{l}\text { At around } 28 \text { weeks } \\
\text { using IADPSG criteria }\end{array}$ & $\begin{array}{l}\text { "Earlier" GDM was mostly } \\
\text { treated with diet and } \\
\text { } \text { lifestyle } \\
\text { modification, with or } \\
\text { without insulin. 19\% of the } \\
\text { 'earlier' GDM group were } \\
\text { not diagnosed and did not } \\
\text { receive any } \\
\text { treatment. } \\
\text { "Recent" all GDM women } \\
\text { received } \\
\text { standardised dietary and } \\
\text { lifestyle advice and } \\
\text { mettormin and/or } \\
\text { insulin if required. }\end{array}$ & Not reported & $<8$ days & $\begin{array}{l}\text { Triceps, subscapular, } \\
\text { flank, quadriceps }\end{array}$ & 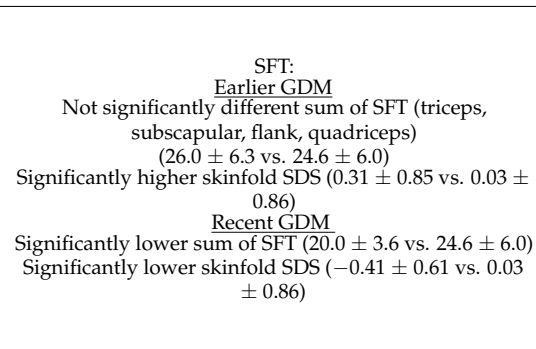 \\
\hline $\begin{array}{c}\text { Buhling, 2012 } \\
\text { Prospective cohort } \\
\text { 2005-2006 } \\
\text { Hamburg, Germany [38] } \\
\text { (additional, data provided by } \\
\text { authors) }\end{array}$ & $\begin{array}{l}\mathrm{NGT}=142 \\
\mathrm{GDM}=30\end{array}$ & $\begin{array}{l}\text { GDM was defined according to the } \\
\text { clinic's guidelines, O'Sullivan } \\
\text { criteria. }\end{array}$ & $\begin{array}{l}\text { Treated with diet or diet + } \\
\text { insulin. }\end{array}$ & Not reported & $<72 \mathrm{~h}$ & $\begin{array}{l}\text { Left anterior iliac spine, at } \\
\text { the lower angle of the left } \\
\text { scapula, at the middle of the } \\
\text { femur, above the left } \\
\text { quadriceps femoris and at } \\
\text { the } \\
\text { middle of the left triceps, } \\
\text { midway between acromion } \\
\text { and olecranon }\end{array}$ & $\begin{array}{c}\text { SFT: } \\
\text { Not significantly different all } 4 \text { sites } \\
\text { triceps, } 4.6 \pm 0.9 \text { vs. } 4.8 \pm 1.5, p=0.67 \\
\text { scapular, } 4.3 \pm 1.41 \text { vs. } 4.1 \pm 0.97, p=0.54 \\
\text { iliac, } 4.4 \pm 1.3 \text { vs. } 4.2 \pm 1.0, p=0.45 \\
\text { femur, } 5.2 \pm 1.8 \text { vs. } 4.7 \pm 1.4, p=0.72\end{array}$ \\
\hline \multicolumn{8}{|c|}{ (b) Studies that measured body composition } \\
\hline & & & & & & 'Dauncy et al. equation $[48]^{\prime}$ & \\
\hline \multirow[t]{2}{*}{$\begin{array}{c}\text { Naf, 2012Prospective } \\
\text { case-control } \\
\text { Joan XXIII University } \\
\text { Hospital, Tarragona, Spain [39] }\end{array}$} & $\begin{array}{l}\mathrm{NGT}=130(46.1 \%) \\
\mathrm{GDM}=84(53.2 \%)\end{array}$ & $\begin{array}{l}\text { National Diabetes Data Group } \\
\text { criteria were used to define GDM } \\
\text { before } 30 \text { weeks }\end{array}$ & $\begin{array}{c}\text { Diet }=48 \\
\text { Diet }+ \text { insulin }=29\end{array}$ & $\begin{array}{c}\text { Target fasting glucose values }<5.3 \\
\mathrm{mmol} / \mathrm{L} \text { and or } 1-\mathrm{h} \text { post-prandial } \\
\text { values }<7.8 \mathrm{mmol} / \mathrm{L} \text {. } \\
\mathrm{GDM} \text { women had higher levels of } \\
\text { fasting glucose4.5 } \pm 0.4 \text { vs. } 4.8 \pm 0.6 \\
\mathrm{mmol} / \mathrm{L}\end{array}$ & $<48 \mathrm{~h}$ & $\begin{array}{l}\text { FM by Dauncy et al. } \\
\text { equation. } \\
\text { Triceps, biceps, subscapular, } \\
\text { and flank skinfold thickness } \\
\text { (data not given) }\end{array}$ & $\begin{array}{l}\text { FM: } \\
\text { Not significantly different } \\
(291 \pm 131 \text { vs. } 318 \pm 133, p=0.198)\end{array}$ \\
\hline & & & & & & $\begin{array}{l}\text { 'Weststrate and Deurenberg } \\
\text { equation [49]' }\end{array}$ & \\
\hline \multirow[t]{2}{*}{$\begin{array}{l}\text { Ubel, 2014 } \\
\text { Cohort } \\
\text { Abteilung fur Geburtshilfe } \\
\text { und Perinatalmedizin } \\
\text { der Frauenklinik, Klinikum } \\
\text { rechts der Isar, Technische } \\
\text { Universititä München } \\
\text { Munich, Germany [41] }\end{array}$} & $\begin{array}{c}\text { Lean NGT }=15 \\
(46.7 \%) \\
\text { Obese NGT }=13 \\
(61.5 \%) \\
\text { Obese GDM }=16 \\
(81.3 \%)\end{array}$ & $\begin{array}{l}\text { Hyperglycaemia and Pregnancy } \\
\text { Outcome (HAPO) criteria }\end{array}$ & $\begin{array}{c}\text { Diet }=7 \\
\text { Insulin treated }=9\end{array}$ & $\begin{array}{c}\text { fasting BGL at 3rd } \\
\text { trimester did not } \\
\text { significantly differ } \\
\text { between the groups and was }<5.1 \\
\mathrm{mmol} / \mathrm{L}\end{array}$ & 1 week & $\begin{array}{l}\text { FM by the equations of } \\
\text { Weststrate } \\
\text { and Deurenberg } \\
\text { Sum of Biceps, triceps, } \\
\text { subscapular, suprailiac }\end{array}$ & $\begin{array}{c}\text { FM: } \\
\text { Significantly higher } \\
\text { compared to lean NGT (694 } \pm 117, \text { vs. } 583 \pm 139, p< \\
0.05) \text {; Not significantly different compared to obese NGT } \\
\text { (694 } \pm 117, \text { vs. } 600 \pm 114, p>0.05) \\
\text { SFT: } \\
\text { Significantly higher compared to lean NGT } \\
\text { (21.6 } 2.4 \text { vs. } 18.9 \pm 3.1) \\
\text { Not significantly different compared to obese NGT (21.6 } \\
\pm 2.4 \text { vs. } 20.3 \pm 2.6)\end{array}$ \\
\hline & & & & & & $\begin{array}{c}\text { 'Catalano et al. equation } \\
{[50]^{\prime}}\end{array}$ & \\
\hline
\end{tabular}


Table 1. Cont.

\begin{tabular}{|c|c|c|c|c|c|c|c|}
\hline $\begin{array}{l}\text { First Author, Year, Study } \\
\text { Design, Time of Data } \\
\text { Collection, Location }\end{array}$ & $\begin{array}{l}\text { Study Groups } n \\
\text { (Males\%) }\end{array}$ & GDM Identification/Definition & Treatment(s) & $\begin{array}{l}\text { Target Blood Glucose Levels (BGLs) } \\
\text { and Level of Glycaemic Control }\end{array}$ & Infants' Age & $\begin{array}{l}\text { Infants' Body Composition } \\
\text { Assessment } \\
\text { Method/SKINFOLD } \\
\text { Thickness Measurements }\end{array}$ & Findings \\
\hline $\begin{array}{l}\text { Aman, 2011 } \\
\text { Case-control } \\
\text { Örebro University Hospital, } \\
\text { Sweden [20] }\end{array}$ & $\begin{array}{l}\mathrm{NGT}=28 \\
\mathrm{GDM}=10\end{array}$ & $\begin{array}{l}\text { 2-h capillary whole-blood glucose } \\
\text { concentration above } 11 \mathrm{mmol} / \mathrm{L}, \\
\text { following a } 75 \text { g OGTT after } 24 \text { th } \\
\text { week of } \\
\text { pregnancy }\end{array}$ & $\begin{array}{l}\text { Dietary adjustments and } \\
\text { multiple pre-meal } \\
\text { insulin injections. }\end{array}$ & $\begin{array}{l}\text { Daily blood glucose target, HbA1c } \\
3.5-5.3 \% \\
\text { Glycaemic control was fairly good, } \\
\text { with mean HbAlc values below the } \\
\text { upper reference limit for healthy } \\
\text { from the 24th to the } 36 \text { th week of } \\
\text { gestation. }\end{array}$ & $<2$ days & $\begin{array}{l}\text { FM by Catalano et al., } \\
\text { equation. } \\
\text { Triceps, subscapular and } \\
\text { abdomen flank SFT }\end{array}$ & $\begin{array}{c}\text { FM: } \\
\text { Significantly higher } \\
(700 \pm 200 \text { vs. } 500 \pm 200, p<0.01) \\
\text { Significantly higher } \\
(17.0 \pm 3.2 \text { vs. } 13.5 \pm 3.5, p<0.01) \\
\text { SFT: } \\
\text { Significantly higher in triceps } \\
(6.6 \pm 1.7 \text { vs. } 5.3 \pm 1.1, p<0.05 \text { ) and subscapular }(6.0 \pm \pm .1, p<0.05) \text { Not significantly different in } \\
2.1 \text { vs. } 4.8 \pm 1.1, p<\text {. } \\
\text { abdominal flank }(5.1 \pm 1.5 \text { vs. } 3.9 \pm 1.0, p>0.05)\end{array}$ \\
\hline $\begin{array}{c}\text { Maple-Brown, } 2019 \\
\text { Longitudinal cohort study } \\
\text { 2011-2017 stre } \\
\text { Northern Territory, Australia } \\
{[45]}\end{array}$ & $\begin{array}{c}\text { Indigenous NGT = } \\
117 \\
\text { Indigenous } \\
\text { GDM/DI } p=278 \\
\text { Non-indigenous } \\
\text { NGT }=118 \\
\text { Non-indigenous } \\
\text { GDM/DIP* }=461\end{array}$ & $\begin{array}{l}\text { GDM were diagnosed by either the } \\
\text { ADIIS guidelines or a universal } 75 \\
\text { gm OGTT and revised glucose cut } \\
\text { points as recommended by the WHO. } \\
\text { DIP, was defined as diabetes first } \\
\text { identified in pregnancy, but with } \\
\text { glucose or HbAlc values higher } \\
\text { glucose than GDM), and identified } \\
\text { from medical } \\
\text { records } \\
\end{array}$ & $\begin{array}{l}\text { Diet only or } \\
\text { Metformin only or } \\
\text { Insulin only or } \\
\text { Metformin and insulin }\end{array}$ & Not reported & $<72 \mathrm{~h}$ & $\begin{array}{l}\text { FM by Catalano et al. } \\
\text { equation. }\end{array}$ & $\begin{array}{c}\text { FM: } \\
\text { Not significantly different } \\
(11.3 \pm 4.2 \text { vs. } 11.5 \pm 3.7, p=0.65) \\
\text { Non-indigenous } \\
\text { Significantly lower } \\
(10.2 \pm 3.7 \text { vs. } 11.5 \pm 3.5, p=0.0006)\end{array}$ \\
\hline \multirow[t]{2}{*}{$\begin{array}{c}\text { Samsuddin, 2020 } \\
\text { Prospective cohort } \\
2014-2017 \\
\text { Tertiary antenatal clinic, Kuala } \\
\text { Lumpur, Malaysia }[47]\end{array}$} & $\begin{array}{c}\text { Obese NGT }=94 \\
\text { Non-obese NGT }= \\
268 \\
\text { GDM }=145 \\
\text { BMI categories } \\
\text { (Asian) } \\
\text { Normal:18.5-22.9 } \\
\mathrm{kg} / \mathrm{m}^{2} ; \text { Overweight: } \\
23-27.4 \mathrm{~kg} / \mathrm{m}^{2} ; \\
\text { Obese: } \geq 27.5 \\
\mathrm{~kg} / \mathrm{m}^{2}\end{array}$ & $\begin{array}{c}\mathrm{FPG} \geq 5.1 \mathrm{mmol} / \mathrm{L} \text { and } / \text { or } 2-\mathrm{h} \\
\text { glucose } \geq 7.8 \mathrm{mmol} / \mathrm{L} \text { atter a } 75 \mathrm{~g} \\
\text { OGTT based on the study centre's } \\
\text { definition and the Malaysian } 2015 \\
\text { Clinical Practice } \\
\text { Guideline }\end{array}$ & $\begin{array}{l}\text { Nutrition therapy. If }>30 \% \text { of } \\
\text { the } \\
\text { self-monitoring of blood } \\
\text { glucose values is } \\
\text { beyond target despite } \\
\text { compliance with medical } \\
\text { nutrition therapy, insulin } \\
\text { therapy is initiated }\end{array}$ & $\begin{array}{l}\text { The glycaemic targets for GDM in } \\
\text { the study } \\
\text { centre: fasting } 3.5-5.1 \mathrm{mmol} / \mathrm{L}, \\
\text { pre-meals } 4.0-5.8 \mathrm{mmol} / \mathrm{L}, 2-\mathrm{h} \\
\text { post-prandiail } 4.0-6.7 \mathrm{mmol} / \mathrm{L} \\
\text { Well-treated GDM } \\
\text { mothers (pre-delivery HbA1c } 5.3 \% \text { ) }\end{array}$ & $<24 \mathrm{~h}$ & $\begin{array}{l}\text { FM by Catalano } \\
\text { et al. equation. } \\
\text { Sum of flank, triceps, } \\
\text { subscapular } \\
\text { SFT }\end{array}$ & $\begin{array}{c}\text { FM: } \\
\text { Not significantly different compared to non-obese NGT } \\
(909 \pm 113 \text { vs. } 924 \pm 149, p>0.05) \text { Significantly lower } \\
\text { compared to obese NGT }(909 \pm 113 \text { vs. } 973 \pm 149, p< \\
0.05) \\
\text { SFT: } \\
\text { Significantly lower sum of SFT (flank, triceps, } \\
\text { subscapular) compared to obese NGT (14.2 } 16.0 \text { vs. } \\
16.1 \pm 5.3, p<0.05) \\
\text { Not significantly different compared to non-obese NGT } \\
(14.2 \pm 3.0 \text { vs. } 14.4 \pm 2.8, p>0.05)\end{array}$ \\
\hline & & & & & & 'TOBEC' & \\
\hline $\begin{array}{c}\text { Okereke, } 2001 \\
\text { Cohort } \\
\text { 1998-2000 } \\
\text { Metro Health Medical } \\
\text { Centre, Cleveland, USA [31] }\end{array}$ & $\begin{array}{l}\mathrm{NGT}=44(58.8 \%) \\
\mathrm{GDM}=34(59.1 \%)\end{array}$ & Carpenter and Coustan criteria & $\begin{array}{c}\text { Diet }=23 \\
\text { Diet }+ \text { insulin }=11\end{array}$ & Not reported & $<48 \mathrm{~h}$ & $\begin{array}{c}\text { TOBEC } \\
\text { paediatric model HP-2 }\end{array}$ & $\begin{array}{l}\text { FM: Significantly higher ( } 480 \pm 210 \text { vs. } 360 \pm 150, p= \\
0 F \text { : Significantly higher (13). } 2 \pm 4.3 \text { vs. } 10.5 \pm 3.8, p= \\
0.01)\end{array}$ \\
\hline
\end{tabular}


Table 1. Cont.

\begin{tabular}{|c|c|c|c|c|c|c|c|}
\hline $\begin{array}{l}\text { First Author, Year, Study } \\
\text { Design, Time of Data } \\
\text { Collection, Location }\end{array}$ & $\begin{array}{l}\text { Study Groups } n \\
\text { (Males } \%)\end{array}$ & GDM Identification/Definition & Treatment(s) & $\begin{array}{l}\text { Target Blood Glucose Levels (BGLs) } \\
\text { and Level of Glycaemic Control }\end{array}$ & Infants' Age & $\begin{array}{l}\text { Infants' Body Composition } \\
\text { Assessment } \\
\text { Method/SKINFOLD } \\
\text { Thickness Measurements }\end{array}$ & Findings \\
\hline \multirow[t]{2}{*}{$\begin{array}{l}\text { Catalano, 2003 } \\
\text { Prospective cohort } \\
\text { 1990-20001regnancy Diabetes } \\
\text { Clinic in Cleveland } \\
\text { Ohio, USA [7] }\end{array}$} & $\begin{aligned} \mathrm{NGT} & =220(54 \%) \\
\mathrm{GDM} & =195(51 \%)\end{aligned}$ & $\begin{array}{l}\text { At } 26 \text { to } 28 \text { weeks using National } \\
\text { Diabetes Data Group criteria }\end{array}$ & $\begin{array}{c}\text { Diet only }=128 \\
\text { Diet }+ \text { insulin }=67\end{array}$ & $\begin{array}{l}\text { Targeted fasting glucose }>5.5 \\
\text { mmol/L and /or } 2 \text { h post-prandial } \\
\text { glucose }>6.7 \mathrm{mmol} / \mathrm{L} \text {. } \\
\text { Women maintained glucose values } \\
\text { within the target range with diet and } \\
\text { exercise }(66 \%) \text {, plus insulin }(34 \%) \text {. }\end{array}$ & $<72 \mathrm{~h}$ & $\begin{array}{l}\text { TOBEC } \\
\text { Tricess and subscapular, } \\
\text { flank, thigh, abdominal SFT }\end{array}$ & 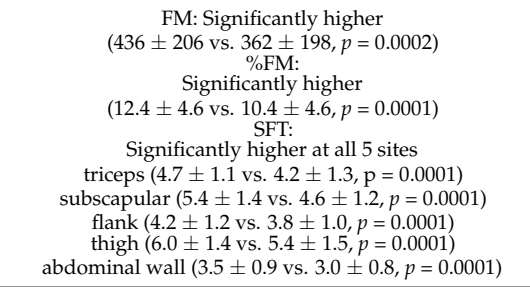 \\
\hline & & & & & & ‘ADP (Pea Pod)' & \\
\hline $\begin{array}{c}\text { Brumbaugh, } 2013 \\
\text { Cross-sectional } \\
\text { University of Colorado } \\
\text { Hospital or Denver Health. } \\
\text { Colorado, USA [40] }\end{array}$ & $\begin{array}{c}\text { Normal NGT }=13 \\
(5.3 .8 \%) \\
\text { Obese/GDM }=12 \\
\text { (66.7\%) } \\
\text { Both groups } \\
\text { matched for } \\
\text { ethnicity }\end{array}$ & $\begin{array}{l}\text { At } 24-28 \text { weeks } \\
\text { using Carpenter and Coustan criteria }\end{array}$ & $\begin{array}{l}2 \text { were diet control, } 10 \text { were } \\
\text { requiring insulin or } \\
\text { glyburide. }\end{array}$ & Not reported & 1-3 weeks & $\begin{array}{l}\text { ADP (Pea Pod) } \\
\text { Sum of triceps and } \\
\text { subscapular SFT }\end{array}$ & $\begin{array}{c}\% \mathrm{FM}: \\
\text { Not significantly different } \\
14.7 \pm 3.0 \mathrm{vs} .13 .1 \pm 5.0, p=0.36 \\
\text { SFT:Significantly higher sum of SFT }(11.7 \pm 1.3 \text { vs. } 9.9 \pm \\
2.0, p=0.01\end{array}$ \\
\hline $\begin{array}{c}\text { Lingwood, 2011 } \\
\text { Prospective cohort } \\
\text { 2009-2010 } \\
\text { Royal Brisbane and Women's } \\
\text { Hospital } \\
\text { Queensian, Australi [221] } \\
\text { (additional data provided by } \\
\text { authors) }\end{array}$ & $\begin{array}{l}\text { NGT }=77(53 \%) \\
\text { GDM }=84(50 \%)\end{array}$ & ADIPS criteria & $\begin{array}{l}\text { Dietary and physical } \\
\text { activity advice. Insulin } \\
\text { treatment was begun if } \\
\text { more than two glucose } \\
\text { measurements exceeded the } \\
\text { target range in } 1 \text { week. }\end{array}$ & $\begin{array}{c}\text { Target BGLs were set } \\
\text { according to current ADIPS } \\
\text { guidelines: } 5.5 \mathrm{mmol} / \mathrm{L} \text { or lower } \\
\text { fasting, and } 7.0 \mathrm{mmol} / \mathrm{L} \text { or lower 2-h } \\
\text { post-prandial. } \\
\text { 80\% met both current fasting and } \\
\text { post-prandial ADIPS targets. } \\
\text { met the lower targets of the } \\
\text { American Diabetes Association }(5.3 \\
\text { and } 6.7 \mathrm{mmol} / \mathrm{L} \text { ) }\end{array}$ & $<6$ days & ADP (Pea Pod) & $\begin{array}{c}\text { FM: } \\
\text { Significantly higher } \\
(413 \pm 192 \text { vs. } 350 \pm 162, p=0.003) \\
\text { \%FM: } \\
\text { Significantly higher } \\
(12.1 \pm 4.3 \text { vs. } 10.1 \pm 4.1, p=0.003)\end{array}$ \\
\hline $\begin{array}{c}\text { Au, 2013 } \\
\text { Cross-sectional } \\
\text { September-Otcober 2010 } \\
\text { Royal Prince-Alfred Hospital } \\
\text { Sydney, Australia [22] }\end{array}$ & $\begin{array}{l}\mathrm{NGT}=532(53 \%) \\
\mathrm{GDM}=67(42 \%)\end{array}$ & ADIPS criteria. & $\begin{array}{l}\text { Dietary and physical } \\
\text { activity advice. Insulin } \\
\text { therapy was commenced } \\
\text { when glycaemic targets } \\
\text { could not be met. }\end{array}$ & $\begin{array}{l}\text { Good glycaemic control was } \\
\text { achieved in no\% of women meeting } \\
\text { both fasting and post-prandial } \\
\text { ADIPS targets }\end{array}$ & $<48 \mathrm{~h}$ & ADP (Pea Pod) & $\begin{array}{c}\text { \%FM: } \\
\text { Not significantly different } \\
7.9 \pm 4.5 \text { vs. } 9.3 \pm 4.3, p=0.018\end{array}$ \\
\hline $\begin{array}{c}\text { Andersson-Hall, } 2018 \\
\text { Longitudinal cohort } \\
\text { 2009-2018 } \\
6 \text { antenatal health units and } \\
\text { Sahlgrenska University } \\
\text { Hospital } \\
\text { Gothenburg, Sweden [44] }\end{array}$ & $\begin{array}{l}\frac{\text { Normal weight group }}{83(50.6 \%)} \\
\frac{\text { Obese group }}{26(65.4 \%)} \\
\frac{\text { GDM group }}{26(38.5 \%)}\end{array}$ & $\begin{array}{l}\text { All pregnant women had non-fasting } \\
\text { blood } \\
\text { glucose measured } \\
\text { regularly throughout pregnancy and } \\
\text { women with an elevated } \\
\text { non-fasting glucose }(>8 \mathrm{mmol} / \mathrm{L}) \\
\text { underwent OGTT. GDM mothers } \\
\text { were identified based on the } \\
\text { European } \\
\text { Association for the Study of Diabetes } \\
\text { criteria, at } 27 \pm 7 \text { gestational weeks. }\end{array}$ & $\begin{array}{l}\text { All } 26 \text { received diet and } \\
\text { lifestyle advice, } 4 \text { received } \\
\text { insulin. }\end{array}$ & Not reported & 4-10 days & ADP (Pea Pod) & $\begin{array}{c}\text { FM: } \\
\text { Normal weight group } \\
\text { Significantly different } \\
(640 \pm 200 \text { vs. 500 } \pm 230, p=0.0034) \\
\text { Obese group } \\
\text { Not significantly different sum of SFT } \\
(640 \pm 200 \text { vs. 580 } 5170, p=0.29) \\
\text { \%FM: } \\
\text { Normal weight group } \\
\text { Significantly different } \\
(16.44 \pm 4.68 \text { vs. } 13.5 \pm \pm .6,6=0.0036) \\
\text { Obese group } p \\
\text { Not significantly different sum of SFT }(16.44 \pm 4.68 \text { vs. } \\
15.23 \pm 3.86, p=0.26)\end{array}$ \\
\hline
\end{tabular}

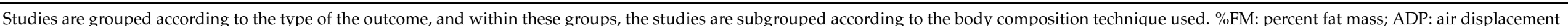

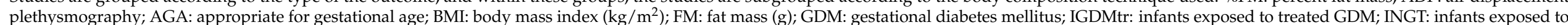

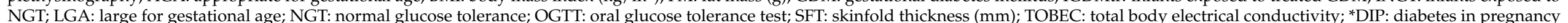

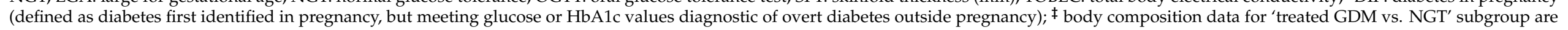
presented as IGDMtr vs. INGT.3.2.1. GDM screening criteria and target blood glucose concentrations. 
Eleven guidelines developed between 1964 and 2014 were used for screening and diagnosing GDM by 22 studies (Table S1). The remaining three studies $[20,33,47]$ used centre-specific criteria for screening and diagnosing GDM. From the 25 studies, 24 used an oral glucose tolerance test (OGTT) to diagnose GDM, and the other [17] used White's classification based on the age of onset and duration of diabetes. Commonly used criteria included Carpenter and Coustan (1982), Australasian Diabetes in Pregnancy Society (ADIPS, 1998), and the International Association of Diabetes and Pregnancy Study Groups (IADPSG, 2011). Seven of the guidelines utilised a screening oral glucose challenge test (OGCT) prior to an OGTT, while other guidelines used only a diagnostic OGTT. Only six of the guidelines tested plasma glucose 3-h post OGCT. The cut-offs for fasting, 1-h, 2-h and 3-h blood glucose ranged between 5.0-7.0 mmol/L, 9.2-11.0 mmol/L, 8.0-9.1 mmol/L and 6.9-8.0 mmol/L, respectively. Seven GDM criteria required two or more abnormal values, while three guidelines required only one abnormal value, for diagnosis of GDM.

Fasting and 2-h post-prandial plasma glucose targets for treated-GDM mothers differed between studies as follows; $5.0 \mathrm{mmol} / \mathrm{L}$ and $6.7 \mathrm{mmol} / \mathrm{L}[33,37], 5.3 \mathrm{mmol} / \mathrm{L}$ and $7.8 \mathrm{mmol} / \mathrm{L}$ [29], $5.3 \mathrm{mmol} / \mathrm{L}$ and $6.7 \mathrm{mmol} / \mathrm{L}$ [25,26], $5.5 \mathrm{mmol} / \mathrm{L}$ and $6.5 \mathrm{mmol} / \mathrm{L}$ [23], $5.5 \mathrm{mmol} / \mathrm{L}$ and $6.7 \mathrm{mmol} / \mathrm{L}[6,19]$, and $5.5 \mathrm{mmol} / \mathrm{L}$ and $7.0 \mathrm{mmol} / \mathrm{L}[11,12,20,24]$. Two studies $[10,32]$ used $\mathrm{HbA1c}$ between $3.5-5.3 \%$ as the mean blood glucose target.

\subsubsection{Treatments Used to Control GDM and Level of Glycaemic Control}

Three studies [17,18,22] used 'diet only', and one study [23] used 'insulin only' to treat GDM while others used combinations of treatments such as 'diet with insulin if required' [10,11,19-21,26-29,31,33,37], 'diet and exercise with insulin, if required' [6,12,32, 34,36]. Metformin, alone or in combination with insulin, was used in two studies [24,35], while glyburide alone or in combination with diet was used in another two studies $[25,30]$. Only $60 \%$ of the studies $(n=15)$ reported the level of glycaemic control in GDM women.

\subsubsection{Adiposity Assessment Techniques Used in the Studies}

Anthropometric and/or body composition information was available in 13 studies, including air displacement plethysmography (ADP) [11,12,30,34], total body electrical conductivity (TOBEC) [6,21], or anthropometric equations proposed by Catalano et al. [10,27,35,37], Dauncy et al. [17,29], and Weststrate and Deurenberg [31]. The most commonly assessed individual SFT sites were triceps and subscapular, and four studies [17,31,33,37] presented the sum of SFT at different sites (data of individual sites were not available).

\subsection{Quality Assessment}

Of the eight criteria listed in the Evidence Project risk of bias tool, two criteria, "(3) pre-post intervention data", and "(8) comparison groups equivalent at baseline on outcome measures", were not applicable for the studies selected for this review (Table 2). All selected studies used non-probability sampling strategies (convenience or self-selected sampling); thus, the criterion "random selection of participants for assessment" was not met by any of them. All studies met the "control or comparison group" criterion. Nineteen studies [6, 11,17,19-21,24-29,31-37] met the criterion "cohort", and except for 1 study [20], all others met the criterion of "follow-up rate of $80 \%$ or more". Only the three randomised control trials (RCT) [24-26] included in the review met the criterion of "(4) random assignment of participants to the intervention". Results of the assessment of the criterion "(7) comparison groups equivalent on sociodemographics" varied across the studies, with the following outcomes: "Equivalent" [20,23,25,30], "Partially Equivalent" [6,21,22,24,26-29,32,34,36], “Not Equivalent" [12,31,37], and “Not Reported" [10,11,17-19,33,35]. 
Table 2. Quality assessment of the studies included in the review, using the Evidence Project risk of bias tool.

\begin{tabular}{|c|c|c|c|c|c|c|c|c|c|c|c|}
\hline \multirow{3}{*}{ First Author } & \multirow{3}{*}{ Year } & \multicolumn{10}{|c|}{ Evidence Project Risk of Bias Tool Items } \\
\hline & & \multirow{2}{*}{$\begin{array}{c}\begin{array}{c}(1) \\
\text { Cohort }\end{array} \\
\text { Judgement }\end{array}$} & \multirow{2}{*}{$\begin{array}{c}\text { (2) } \\
\begin{array}{c}\text { Control or } \\
\text { Comparison } \\
\text { Group }\end{array} \\
\text { Judgement } \\
\end{array}$} & \multirow{2}{*}{$\begin{array}{c}\text { (3) } \\
\begin{array}{c}\text { Pre/Post } \\
\text { Intervention } \\
\text { Data }\end{array} \\
\text { Judgement } \\
\end{array}$} & \multirow{2}{*}{$\begin{array}{c}\text { (4) } \\
\text { Random } \\
\text { Assignment of } \\
\text { Participants to } \\
\text { the Intervention } \\
\text { Judgement } \\
\end{array}$} & \multirow{2}{*}{$\begin{array}{c}5) \\
\text { Random } \\
\begin{array}{c}\text { Selection of } \\
\text { Participants for } \\
\text { Assessment }\end{array} \\
\text { Judgement } \\
\end{array}$} & \multicolumn{2}{|c|}{$\begin{array}{c}(6) \\
\text { Follow-Up Rate of } 80 \% \text { or More }{ }^{a}\end{array}$} & \multicolumn{2}{|c|}{$\begin{array}{c}(7) \\
\text { Comparison Groups Equivalent on } \\
\text { Sociodemographic }^{b}\end{array}$} & \multirow{2}{*}{ 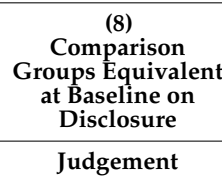 } \\
\hline & & & & & & & Judgement & Follow-U p Rate & Judgement & Comment & \\
\hline $\begin{array}{c}\text { Enzi } \\
\text { Stevenson }\end{array}$ & $\begin{array}{l}1980 \\
1991\end{array}$ & Yes & $\begin{array}{l}\text { Yes } \\
\text { Yes }\end{array}$ & No & NA & No & Yes & $87.5 \%$ & NR & \multirow{3}{*}{$\begin{array}{c}\text { Ethnicity and sex not significantly } \\
\text { different }\end{array}$} & NA \\
\hline $\begin{array}{l}\text { Stevenson } \\
\text { Vohr }\end{array}$ & $\begin{array}{l}1991 \\
1995\end{array}$ & $\begin{array}{l}\text { No } \\
\text { Yes }\end{array}$ & $\begin{array}{l}\text { Yes } \\
\text { Yes }\end{array}$ & $\begin{array}{l}\text { No } \\
\text { No }\end{array}$ & $\begin{array}{l}\text { NA } \\
\text { NA }\end{array}$ & $\begin{array}{l}\text { No } \\
\text { No }\end{array}$ & $\begin{array}{l}\text { NA } \\
\text { Yes }\end{array}$ & $100 \%$ & $\begin{array}{l}\mathrm{NR} \\
\mathrm{NR}\end{array}$ & & $\begin{array}{l}\text { NA } \\
\text { NA }\end{array}$ \\
\hline Simmons & 1997 & Yes & Yes & No & NA & No & No & $57 \%$ & Yes & & NA \\
\hline Okereke & 2001 & Yes & Yes & No & NA & No & Yes & $100 \%$ & Partial & $\begin{array}{l}\text { Sex not significantly different, } \\
\text { ethnicity significantly different }\end{array}$ & NA \\
\hline Catalano & 2003 & Yes & Yes & No & NA & No & Yes & \multirow[t]{3}{*}{$100 \%$} & Partial & \multirow{3}{*}{$\begin{array}{c}\text { Ethnicity significantly different, } \\
\text { sex not significantly different } \\
\text { Sex not significantly different } \\
\text { Sex and ethnicity not significantly } \\
\text { different }\end{array}$} & NA \\
\hline $\mathrm{Ng}$ & 2004 & No & Yes & No & NA & No & NA & & Partial & & NA \\
\hline Westgate & 2006 & No & Yes & No & NA & No & NA & & Yes & & NA \\
\hline Rowan & $\begin{array}{l}2008 \\
2009\end{array}$ & $\begin{array}{l}\text { Yes } \\
\text { Yes }\end{array}$ & $\begin{array}{l}\text { Yes } \\
\text { Yes }\end{array}$ & No & $\begin{array}{l}\text { Yes } \\
\text { Yes }\end{array}$ & No & $\begin{array}{l}\text { Yes } \\
\text { Yes }\end{array}$ & $97.6 \%$ & Partial & Ethnicity not significantly different & NA \\
\hline Landon & 2009 & Yes & $\begin{array}{l}\text { Yes } \\
\text { Yes }\end{array}$ & $\begin{array}{l}\text { No } \\
\text { No }\end{array}$ & $\begin{array}{l}\text { Yes } \\
\text { Yes }\end{array}$ & $\begin{array}{l}\text { No } \\
\text { No }\end{array}$ & $\begin{array}{l}\text { Yes } \\
\text { Yes }\end{array}$ & $\begin{array}{l}82.8 \% \\
93.9 \%\end{array}$ & $\begin{array}{l}\text { Yes } \\
\text { Partial }\end{array}$ & \multirow{3}{*}{ Ethnicity not significantly different } & $\begin{array}{l}\text { NA } \\
\text { NA }\end{array}$ \\
\hline Aman & 2011 & No & Yes & No & NA & No & NA & & NR & & NA \\
\hline Lingwood & 2011 & Yes & Yes & No & NA & No & Yes & $100 \%$ & NR & & NA \\
\hline Naf & 2011 & Yes & Yes & No & NA & No & Yes & $100 \%$ & Partial & \multirow{3}{*}{$\begin{array}{c}\text { Sex not significantly different } \\
\text { Sex not significantly different } \\
\text { Significant difference in maternal } \\
\text { ethnicity }\end{array}$} & NA \\
\hline Schaefer-Graf & 2011 & Yes & Yes & No & NA & No & Yes & $100 \%$ & Partial & & NA \\
\hline $\mathrm{Au}$ & 2012 & No & Yes & No & NA & No & NA & & No & & NA \\
\hline Brumbaugh & 2013 & No & Yes & No & NA & No & NA & & Yes & \multirow{3}{*}{$\begin{array}{c}\text { Ethnicity not significantly different } \\
\text { Sex and ethnicity not significantly } \\
\text { different } \\
\text { Sex significantly different }\end{array}$} & NA \\
\hline Ubel & 2014 & Yes & Yes & No & NA & No & Yes & $100 \%$ & No & & NA \\
\hline Mitanchez & 2017 & yes & Yes & No & NA & No & Yes & $90.3 \%$ & NR & & NA \\
\hline Kara & 2017 & Yes & Yes & No & NA & No & Yes & $100 \%$ & Partial & Sex not significantly different & NA \\
\hline Andersson-Hall & 2018 & Yes & Yes & No & NA & No & Yes & $83 \%$ & Partial & Sex not significantly different & NA \\
\hline Maple-Brown & 2019 & Yes & Yes & No & NA & No & Yes & $100 \%$ & NR & & NA \\
\hline Prentice & 2019 & Yes & Yes & No & NA & No & $\mathrm{NR}$ & & Partial & Sex not significantly different & NA \\
\hline Samsuddin & 2020 & Yes & Yes & No & NA & No & Yes & $100 \%$ & No & Ethnicity significantly different & NA \\
\hline
\end{tabular}

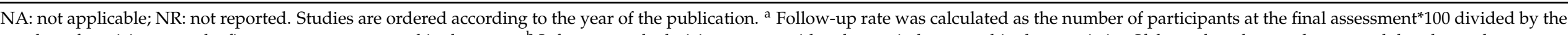

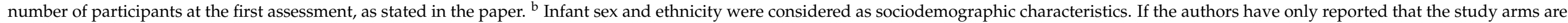

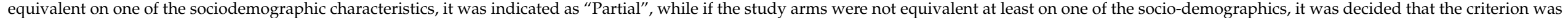
not met ("No"). 


\subsection{Effects of Treatments for GDM on Infant Adiposity}

\subsubsection{Treated GDM vs. No Treatment for GDM}

One RCT [36] investigated whether treatment for GDM normalised infant adiposity at birth. In this study of 958 GDM women (485 treated vs. 473 no treatment), mean FM in infants of GDM mothers who received the treatment of diet therapy $(n=427)$ and insulin, if required $(n=36)$, was significantly lower $(p=0.003)$ than that of control infants whose mothers received usual prenatal care $(427 \pm 198 \mathrm{~g}$ vs. $464 \pm 222 \mathrm{~g})$.

\subsubsection{Different Treatment Regimens for GDM}

Two studies $[7,30]$ compared the effects of treating GDM with lifestyle modification alone vs. lifestyle modification plus insulin, on infant birth measurements. A small study [30] with a sample size of 20, found no significant differences in mean subscapular SFT, between GDM exposed infants whose mothers were treated with 'diet alone' and 'diet with insulin'. A comparatively larger study [7], with a sample size of 195, revealed that compared to 'diet and exercise only', infants whose mothers were treated with 'diet, exercise and insulin' had higher FM (492 $\pm 215 \mathrm{~g}$ vs. $407 \pm 196 \mathrm{~g}, p=0.006)$ and \%BF $(13.6 \% \pm 4.6 \%$ vs. $11.7 \pm 4.5 \%, p=0.007)$. These effects persisted even after adjusting for gestational age, maternal pregravid weight and parity. Two RCTs [34,35] investigated the difference in adiposity in infants of GDM mothers, who were treated with pharmacological treatments for GDM. Rowan et al. [34] compared treating GDM women with metformin (with supplemental insulin, if required, $n=363)$ to treatment with insulin alone $(n=370)$ and reported that triceps (5.2 \pm 1.6 vs. $5.1 \pm 1.2, p=0.30)$ and subscapular $(5.2 \pm 1.5$ vs. $5.2 \pm 1.3, p=0.60)$ SFT $(\mathrm{mm})$ were not significantly different between the groups. Lain et al. [35] compared insulin $(n=41)$ with glyburide $(n=41)$, and found no significant differences in mean triceps SFT ( $3.9 \pm 0.7$ vs. $3.9 \pm 0.9, p=0.89)$, subscapular SFT $(4.1 \pm 1.0$ vs. $4.5 \pm 1.3, p=0.10)$, suprailiac SFT ( $2.1 \pm 0.6$ vs. $2.1 \pm 0.6, p=0.85)$, thigh SFT $(5.1 \pm 1.2$ vs. $5.4 \pm 1.7, p=0.28), \mathrm{FM}(370 \pm 167$ vs. $473 \pm 278, p=0.06)$ or $\% \mathrm{FM}(11.2 \pm 4.2$ vs. $12.8 \pm 5.7, p=0.18)$. None of the studies compared 'lifestyle modification alone' with 'pharmaceutical interventions'.

\subsubsection{Treated GDM vs. NGT}

Fat Mass (FM)

Ten studies $[7,20,21,27,31,37,39,41,44,47]$ reported the effect of treated GDM compared to NGT on infant FM. Overall, infants born to mothers with treated GDM (IGDMtr) had significantly higher FM (mean difference, 95\% confidence interval: $68.46 \mathrm{~g}, 29.91$ to 107.01) than infants born to NGT mothers (INGT); (Figure 2).

\begin{tabular}{|c|c|c|c|c|c|c|c|c|c|c|c|c|c|}
\hline \multirow[b]{2}{*}{ Study or Subgroup } & \multicolumn{3}{|c|}{ IGDMtr } & \multicolumn{3}{|c|}{ INGT } & \multicolumn{3}{|c|}{ Mean Difference } & \multirow{2}{*}{\multicolumn{4}{|c|}{$\begin{array}{c}\text { Mean Difference } \\
\text { IV, Random, } 95 \% \mathrm{Cl}\end{array}$}} \\
\hline & Mean $[g]$ & SD [g] & Total & Mean [g] & SD $[g]$ & Total & Weight & IV, Random, $95 \% \mathrm{Cl}$ & Year & & & & \\
\hline Enzi 1980 [27] & 539 & 49 & 17 & 386 & 22 & 17 & $12.3 \%$ & $153.00[127.47,178.53]$ & 1980 & & & $=$ & \\
\hline Okereke 2002 [31] & 480 & 210 & 34 & 360 & 150 & 44 & $8.1 \%$ & $120.00[36.65,203.35]$ & 2002 & & & & \\
\hline Catalano 2003 [7] & 436 & 206 & 195 & 362 & 198 & 220 & $11.4 \%$ & $74.00[35.01,112.99]$ & 2003 & & & $\rightarrow$ & \\
\hline Schaefer-Graf 2011 [37] & 433 & 14 & 150 & 381 & 13 & 190 & $12.9 \%$ & $52.00[49.10,54.90]$ & 2011 & & & - & \\
\hline Lingwood 2011 [21] & 413 & 192 & 84 & 350 & 162 & 77 & $10.3 \%$ & $63.00[8.27,117.73]$ & 2011 & & & & \\
\hline Aman 2011 [20] & 700 & 200 & 10 & 500 & 200 & 28 & $4.6 \%$ & $200.00[55.59,344.41]$ & 2011 & & & & \\
\hline Naf 2012 [39] & 291 & 131 & 84 & 318 & 133 & 130 & $11.6 \%$ & $-27.00[-63.16,9.16]$ & 2012 & & $\rightarrow$ & & \\
\hline Ubel 2014 [41] & 694 & 117 & 16 & 619 & 132 & 28 & $8.7 \%$ & $75.00[-0.35,150.35]$ & 2014 & & & & \\
\hline Anderson 2019 [44] & 640 & 200 & 26 & 520 & 230 & 109 & $7.7 \%$ & $120.00[31.83,208.17]$ & 2019 & & & & \\
\hline Samsudin 2020 [47] & 909 & 113 & 145 & 937 & 131 & 362 & $12.4 \%$ & $-28.00[-50.81,-5.19]$ & 2020 & & - & & \\
\hline Total $(95 \% \mathrm{Cl})$ & & & 761 & & & 1205 & $100.0 \%$ & $68.46[29.91,107.01]$ & & & & & \\
\hline $\begin{array}{l}\text { Heterogeneity: } \mathrm{Tau}^{2}=29 \\
\text { Test for overall effect: } Z=\end{array}$ & $\begin{array}{l}\text { 6.33; } \mathrm{Chi}^{2}= \\
.48(\mathrm{P}=0.0\end{array}$ & $\begin{array}{l}\text { 136.30, } \\
005)\end{array}$ & $d f=9$ & $(P<0.000$ & 11); $\left.\right|^{2}=$ & & & & & -500 & $\begin{array}{l}-250 \\
\text { Higher in INGT }\end{array}$ & $\begin{array}{c}250 \\
\text { Higher in IGDMtr }\end{array}$ & 500 \\
\hline
\end{tabular}

Figure 2. Forest plot comparing fat mass (g) in infants exposed to treated gestational diabetes mellitus (IGDMtr) and infants exposed to normal glucose tolerance (INGT). 


\section{Percent Fat Mass (\%FM)}

Nine studies [7,20-22,27,31,40,44,45] investigated the effect of treated GDM compared to NGT on infant \%FM. In the pooled result, \%FM (1.98\%, 0.54 to 3.42$)$ in IGDMtr was significantly higher than INGT (Figure 3).

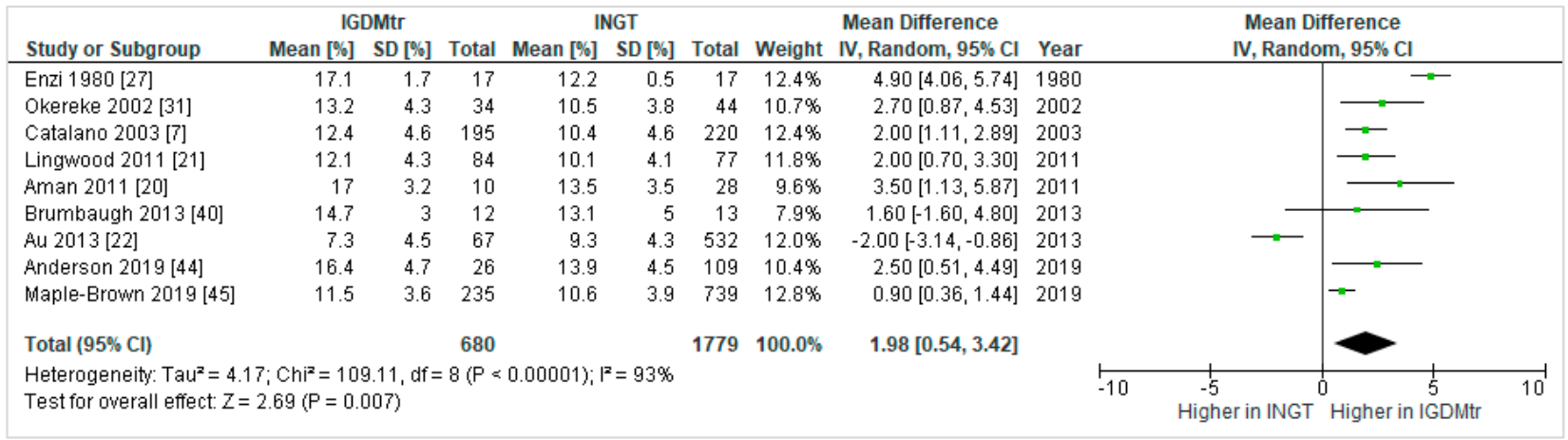

Figure 3. Forest plot comparing percent fat mass (\%) in infants exposed to treated gestational diabetes mellitus (IGDMtr) and infants exposed to normal glucose tolerance (INGT).

\section{Skinfold Thickness (SFT)}

The number of studies that reported SFT at individual skinfold sites were as follows: triceps $=8[7,20,28,29,33,38,42,46] ;$ subscapular $=7[7,20,29,33,38,42,46]$; flank $=3[7,20,46]$; and abdominal $=2[7,29]$. None of the comparisons of skinfold sites were significantly different between IGDMtr and INGT infants in the pooled results; triceps: $0.14 \mathrm{~mm},-0.35$ to 0.63 (Figure $4 \mathrm{a}$ ); subscapular: $0.44 \mathrm{~mm},-0.15$ to 1.02 (Figure $4 \mathrm{~b}$ ); flank: $0.04 \mathrm{~mm},-1.35$ to 1.44 (Figure 4c) and abdominal: $0.33 \mathrm{~mm},-0.06$ to 0.72 (Figure $4 \mathrm{~d}$ ). Several other SFT sites, i.e., biceps [42], quadriceps [46], suprailiac [29], iliac [38], femur [38], thigh [7] and calf [29], were reported in single studies, and therefore, a meta-analysis could not be performed.

Four studies compared IGDMtr vs. INGT using sum of SFT at different body sites, therefore they were not included in the meta-analysis. Of those, two reported that the sum of SFT at 'triceps and subscapular' [40] and 'subscapular, subcostal, tricipital and crural' [27] was significantly higher in IGDMtr. Another study [47] reported that the sum of SFT at 'flank, triceps and subscapular' was not significantly different between IGDMtr and INGT. A study [32] in which data were not normally distributed presented median and interquartile range and reported that triceps $(4.8 \mathrm{~mm}(4.2-5.1)$ vs. $4.7 \mathrm{~mm}(4.1-5.5))$ and subscapular (4.8 $\mathrm{mm}(4.3-5.3)$ vs. $4.8 \mathrm{~mm}(4.1-5.3))$ SFT were not significantly different between the two infant groups.

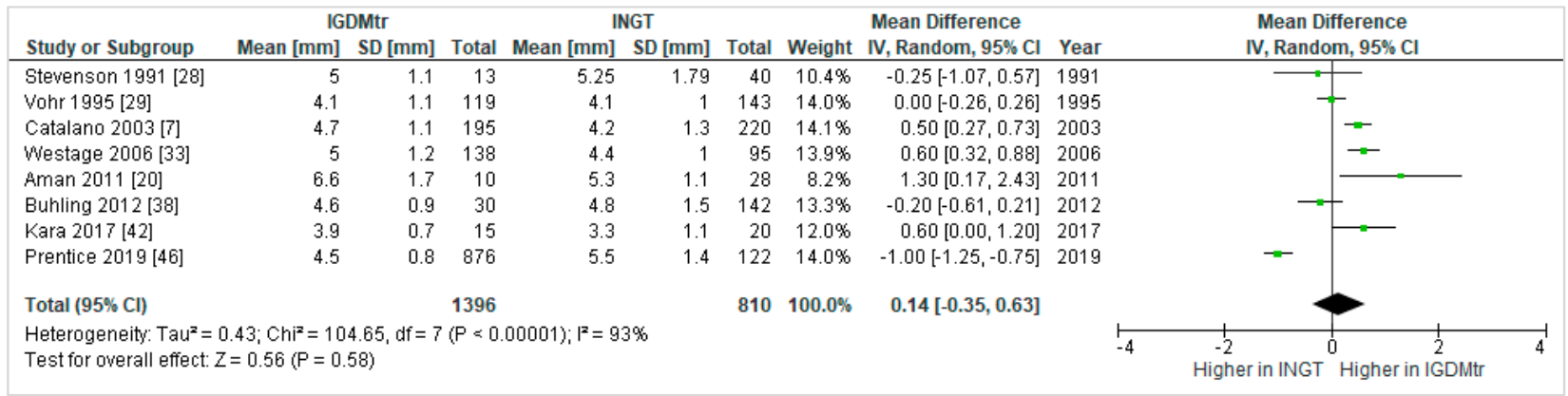

(a)

Figure 4. Cont. 


\begin{tabular}{|c|c|c|c|c|c|c|c|c|c|c|c|c|}
\hline \multirow[b]{2}{*}{ Study or Subgroup } & \multicolumn{2}{|c|}{ IGDMtr } & \multicolumn{3}{|c|}{ INGT } & \multirow[b]{2}{*}{ Total } & \multirow[b]{2}{*}{ Weight } & \multirow{2}{*}{$\begin{array}{l}\text { Mean Difference } \\
\text { IV, Random, } 95 \% \mathrm{Cl}\end{array}$} & \multirow[b]{2}{*}{ Year } & \multirow{2}{*}{\multicolumn{2}{|c|}{$\begin{array}{c}\text { Mean Difference } \\
\text { IV, Random, } 95 \% \mathrm{Cl}\end{array}$}} & \\
\hline & Mean [mm] & SD [mm] & Total & Mean $[\mathrm{mm}]$ & $\mathrm{SD}[\mathrm{mm}]$ & & & & & & & \\
\hline Vohr 1995 [29] & 4.7 & 1.5 & 119 & 4.6 & 1.3 & 143 & $15.6 \%$ & $0.10[-0.24,0.44]$ & 1995 & & & \\
\hline Catalano $2003[7]$ & 5.4 & 1.4 & 195 & 4.6 & 1.2 & 220 & $16.0 \%$ & $0.80[0.55,1.05]$ & 2003 & & $=$ & \\
\hline Westage 2006 [33] & 5.6 & 1.6 & 138 & 4.4 & 1 & 95 & $15.6 \%$ & $1.20[0.87,1.53]$ & 2006 & & -- & \\
\hline Aman 2011 [20] & 6 & 2.1 & 10 & 4.8 & 1.1 & 28 & $8.7 \%$ & $1.20[-0.16,2.56]$ & 2011 & & & \\
\hline Buhling 2012 [38] & 4.3 & 1.4 & 30 & 4.1 & 1 & 142 & $14.5 \%$ & $0.20[-0.33,0.73]$ & 2012 & - & 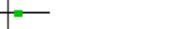 & \\
\hline Kara 2017 [42] & 3.8 & 0.8 & 15 & 3.4 & 1.2 & 20 & $13.6 \%$ & $0.40[-0.26,1.06]$ & 2017 & & & \\
\hline Prentice 2019 [46] & 4.8 & 1 & 122 & 5.3 & 1.3 & 876 & $16.1 \%$ & $-0.50[-0.70,-0.30]$ & 2019 & $\rightarrow$ & & \\
\hline Total $(95 \% \mathrm{Cl})$ & & & 629 & & & 1524 & $100.0 \%$ & $0.44[-0.15,1.02]$ & & & & \\
\hline $\begin{array}{l}\text { Heterogeneity: Tau } \\
\text { Test for overall effect }\end{array}$ & $\begin{array}{l}0.54 ; \mathrm{Chi}^{2}=1 \\
Z=1.47(\mathrm{P}=\end{array}$ & $\begin{array}{l}07.20, d f= \\
0.14)\end{array}$ & $6(\mathrm{P}<0$ & $0.00001) ;\left.\right|^{2}=$ & $94 \%$ & & & & & $\begin{array}{c} \\
-2 \\
\text { Higher in INGT }\end{array}$ & $\begin{array}{l}0 \\
0\end{array}$ & 4 \\
\hline
\end{tabular}

(b)

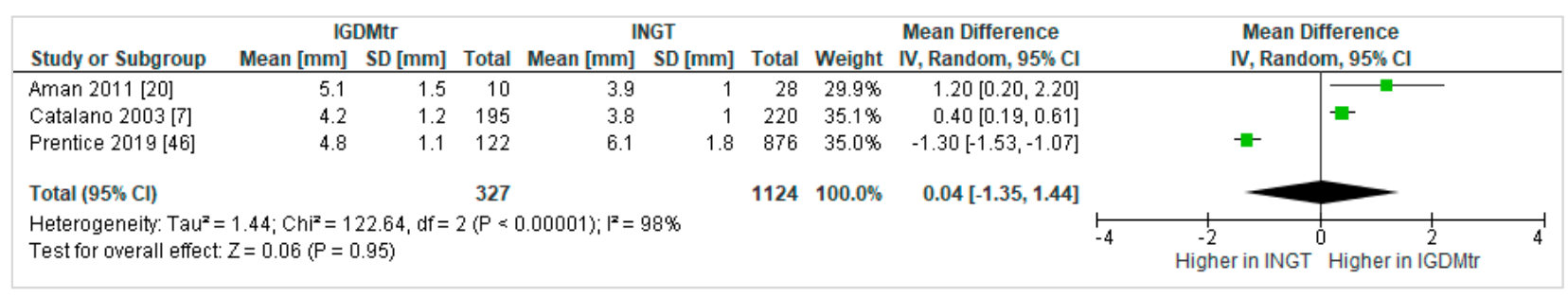

(c)

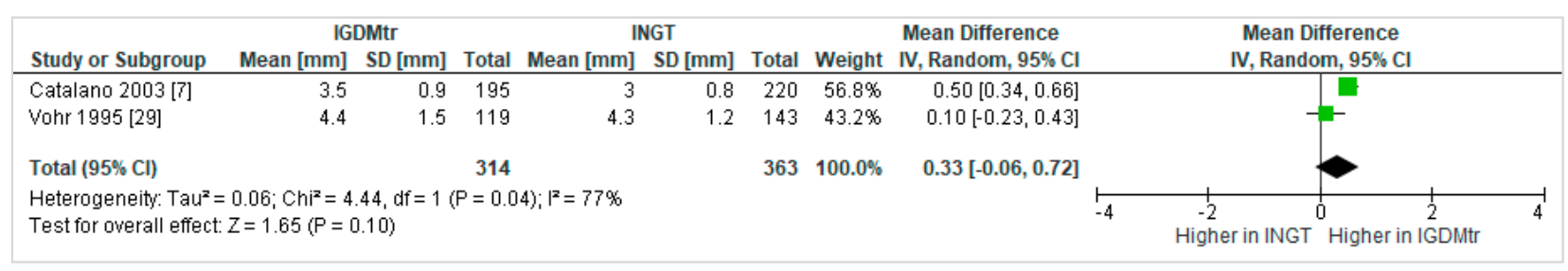

(d)

Figure 4. Forest plots comparing skinfold thickness $(\mathrm{mm})$ at triceps (a), subscapular (b), flank (c) and abdomen (d) in infants exposed to treated gestational diabetes mellitus (IGDMtr) and infants exposed to normal glucose tolerance (INGT).

Heterogeneity between the Studies That Compared GDMtr vs. NGT

A high proportion of the observed heterogeneity in all the meta-analyses (as indicated by an $\mathrm{I}^{2}$ statistic $>90 \%$ ) was due to underlying between-study differences [51]. We considered whether the GDM mothers achieved good glycaemic control with the treatments as one of the potential sources of heterogeneity. However, the information on the level of glycaemic control in GDM mothers was not reported in $40 \%$ of studies [27,30-33,38,40,43,45,46]. Therefore, the studies in which the authors stated that the mothers achieved good glycaemic control were separated from other studies, to see if the achievement of good glycaemic control mediated the relationship between GDM and infant adiposity. The test for subgroup differences indicated that there was no statistically significant subgroup effect of studies indicating GDM mothers achieving good glycaemic control on infant FM $(p=0.76)$, \%FM $(p=0.15)$, triceps $(p=0.34)$ and subscapular SFT $(p=0.73)$.

The test for subgroup differences in 'pre-2010' vs. 'post-2010' studies showed a statistically significant subgroup effect on FM ( $p=0.03$, Figure S2) and \%FM ( $p=0.02$, Figure S3). There was no significant difference in FM and \%FM between IGDMtr and INGT in 'post-2010' studies, whereas, in 'pre-2010' studies, FM and \%FM were significantly higher in IGDMtr compared to their counterparts. Further, subgroup analyses by infant body composition assessment technique were performed for infant FM and \%FM. There was no significant effect ( $p=0.28$ ) of body composition technique on infant FM (Figure S4). Subgroup difference in \%FM was significant $(p<0.00001)$; however, the number of studies and participants who contributed to subgroups were considerably different (Figure S5). 
$\%$ FM measured with ADP $(0.93 \%,-1.61$ to 3.47$)$ or the Catalano et al. equation $(1.93 \%$, -0.56 to 4.43 ) did not significantly differ between IGDMtr and INGT. \%FM measured by TOBEC $(2.13 \%, 1.34$ to 2.93$)$ or the Dauncy et al. equation $(4.90 \%, 4.06$ to 5.74$)$ was higher in IGDMtr. Leave-one-out sensitivity analysis demonstrated that removing the studies that had used the Catalano equation or TOBEC changed the overall effect for \%FM to statistical non-significance. Of note, from the four studies that used ADP [21,22,40,44], three $[21,22,44]$ affirmed good glycaemic control in mothers. Sensitivity analysis performed after removing the study [40] with no data on glycaemic control did not change the pooled result for the ADP subgroup. Moreover, leave-one-GDM-criteria-out sensitivity testing for FM and \%FM did not show significant changes in the pooled effects. Specifically, the sensitivity analysis for White's classification, which is different from other criteria that use an OGTT, did not significantly change the overall results for infant FM and \%FM (Figure S6).

\section{Discussion}

We performed a systematic review and a meta-analysis of published studies (irrespective of the study designs) reporting adiposity in infants exposed to GDM controlled with therapeutic interventions. Treatments for GDM lowered newborn adiposity compared to no treatment, and there were no significant differences in adiposity in IGDMtr according to the mode of therapy; however, the evidence was insufficient due to the low number of available studies. IGDMtr had higher FM and \%FM compared to INGT, but there was no significant difference in subcutaneous adiposity as measured by SFT.

Accelerated fat deposition in the foetus of GDM women can be reduced by strict glycaemic control [36]. Most women with GDM can control blood glucose with lifestyle changes such as diet modification, and increased physical activity; however, approximately one-third of women may require additional pharmacological treatments [14]. Oral diabetic medication is widely accepted by pregnant women in contrast to insulin because of easier storage, administration and lower cost [52], but unlike insulin, both metformin and glyburide cross the placenta [53]. Additionally, meta-analyses of risks and benefits of using insulin, metformin and glyburide in GDM women requiring drug treatment have shown that glyburide is inferior to both insulin and metformin, resulting in higher birth weights and increased risk of macrosomia, while metformin is associated with more preterm births than insulin [54]. On the other hand, insulin can bind to its specific receptor (in the placenta) to activate its signalling pathways; thus, insulin treatment still may have effects on placental and foetal growth [55]. One of the studies included in our review [7] reported that treatment with insulin in addition to lifestyle modification significantly increased the FM and \%FM in IGDMtr as opposed to lifestyle intervention alone; however, the authors speculated that there might have been a confounding effect of other maternal factors associated with increased infant adiposity, as the former group of mothers (i.e., those who also received insulin) were characterised with higher pre-pregnancy weight and parity than their counterparts. Moreover, metformin and glyburide can impact foetal growth in opposite ways [56,57]. Glyburide controls maternal hyperglycaemia by stimulating insulin production. When glyburide is transported to the foetus through the placenta, it may also increase insulin secretion by the foetal pancreas that results in foetal overgrowth [56]. On the other hand, metformin inhibits glucose and amino acid transportation from the mother to the developing foetus through the placenta [57], which may cause foetal undergrowth. Despite this, the findings of the two RCTs included in our review [34,35] suggested that the effects of metformin, glyburide or insulin on infant adiposity were not significantly different; nonetheless, more studies are required for definitive conclusions. As reported in two recent systematic reviews, although there are no significant differences in body composition at birth [58], children exposed to metformin in utero show accelerated postnatal growth, compared to those exposed to insulin [59]. Therefore, tracking body composition trajectory of children exposed to pharmacological interventions in utero should be a research priority. 
Our meta-analysis shows that treatments for GDM normalise newborns' subcutaneous fat measured by SFT, but not overall adiposity measured by FM and \%FM. These findings suggest that the phenotype of the IGDMtr may be distinguished with increased internal adiposity. Increased intra-abdominal adiposity is associated with several metabolic disorders, while superficial subcutaneous adiposity may exert a protective effect [60]. Furthermore, exposure to excess fuels in the gestational environment may lead to increased hepatic fat deposition in the foetus, which possibly plays a role in the development of nonalcoholic liver disease in children [61]. On the other hand, the accuracy of SFT measurements is dependent on the skills of the measurer, and the adiposity prediction equations with SFT are highly specific to the infant population that the data were derived from [62]. Thus, differentiating adipose tissue compartments with more reliable objective techniques and assessing hepatic fat deposition in IGDMtr and INGT at birth is important to identify these differences and any effects of GDM treatments. Comparing different adiposity compartments was beyond the scope of the current review, and such studies are very limited. Two small studies $[40,63]$ reported that there were no significant differences in $\% F M$, subcutaneous fat $\left(\mathrm{cm}^{3}\right)$ and intra-abdominal fat/length $\left(\mathrm{cm}^{2}\right)$ at 1-3 weeks [40], and in total adipose tissue $\left(\mathrm{cm}^{3}\right)$, subcutaneous adipose tissue $\left(\mathrm{cm}^{3}\right)$, internal abdominal adipose tissue $\left(\mathrm{cm}^{3}\right)$ at 1-2 weeks [63] in IGDMtr and INGT infants. Intriguingly, one study reported a significant increase in intrahepatocellular lipid content in IGDMtr compared to INGT, while the other did not detect such a difference. However, glycaemic control was not described in the former study, whereas $\sim 80 \%$ of mothers in the latter study had good glycaemic control with a mean third-trimester $\mathrm{HbA} 1 \mathrm{c}$ level of $5.3 \%$.

There were no significant differences in \%FM in IGDMtr and INGT in studies 'post$2010^{\prime}$ or when newborn overall adiposity was measured with ADP. These findings may be attributed to more intensive management of hyperglycaemia in the 'post-2010' period. Moreover, our findings highlight the importance of using more accurate and reliable objective infant body composition techniques such as ADP. The high degree of between-study heterogeneity may have arisen from the use of a wide variety of GDM diagnostic criteria, differences in the severity of hyperglycemia and level of glycaemic control, and confounding effects of maternal obesity, ethnicity, gestational weight gain, smoking, gestational age, infants' sex and age at the investigation. Future studies should adopt universal criteria for the diagnosis of GDM, use reliable body composition assessment techniques such as ADP, and report the treatments and level of glycaemic control in GDM mothers throughout the pregnancy to enable robust conclusions on the association between GDM and newborn adiposity.

To our knowledge, the current review is the first to simultaneously evaluate studies reporting adiposity in newborns exposed to treated GDM vs. no treatment, different treatment regimens for GDM, and treated GDM vs. NGT. Adiposity in infants exposed to GDM compared to NGT has been investigated in a subgroup analysis of a previous systematic review [64] that examined the literature focused on the effect of all types of maternal diabetes. The authors found higher FM, \%FM, triceps SFT and subscapular SFT in GDM-exposed infants compared to NGT; however, in some of the studies included in their meta-analysis (e.g., HAPO Study [11]), mothers were not treated. Other strengths of our study include the search of the literature in five major databases, investigation of differences in SFT sites such as abdominal and flank regions in addition to commonly reported triceps and subscapular measures, and investigation of potential sources of heterogeneity via subgroup and sensitivity analyses. Limitations of our study were that we only included studies published in English, excluded studies in which GDM status was self-reported by mothers where no information was reported on the use of treatments for glycaemic control, and considered only the most common measures of adiposity, i.e., FM, \%FM and SFT, for comparison purposes. 


\section{Conclusions}

Irrespective of the therapeutic strategy, treatment for GDM appears to reduce excess adiposity characteristic for newborns exposed to untreated GDM, but the evidence is limited. Due to the potential effects of oral hypoglycaemic medications on foetal growth, further studies on the impact of different GDM therapies on newborn adiposity are also warranted. Despite the significant heterogeneity found between the studies, our metaanalysis revealed higher overall adiposity (as measured with FM and \%FM) but similar subcutaneous adiposity (as measured with SFT) in IGDMtr compared to INGT, suggesting that higher adiposity in IGDMtr may be due to excess fat accrual in internal adipose tissue. Future studies should distinguish adipose tissue distribution of IGDMtr and INGT with sufficient power to confirm these differences.

Supplementary Materials: The following are available online at https://www.mdpi.com/2077-038 3/10/4/835/s1. Table S1: Diagnostic Criteria for gestational diabetes mellitus (GDM) used by the studies included in the review; Figure S1: Search strategy in Medline via Ovid; Figure S2: Forest plot comparing fat mass (g) in infants exposed to treated gestational diabetes mellitus (IGDM) and infants exposed to normal glucose tolerance (INGT) by subgroup analysis of time of the study; 'pre-2010' vs. 'post-2010'; Figure S3: Forest plot comparing percent fat mass (\%) in infants exposed to treated gestational diabetes mellitus (IGDM) and infants exposed to normal glucose tolerance (INGT) by subgroup analysis of time of the study; 'pre-2010' vs. 'post-2010'; Figure S4: Forest plot comparing fat mass (g) in infants exposed to treated gestational diabetes mellitus (IGDM) and infants exposed to normal glucose tolerance (INGT) by subgroup analysis of infant body composition assessment technique; Figure S5: Forest plot comparing percent fat mass (\%) in infants exposed to treated gestational diabetes mellitus (IGDM) and infants exposed to normal glucose tolerance (INGT) by subgroup analysis of infant body composition assessment technique. Figure S6: Forest plot comparing percent fat mass (a) and percent fat mass (b) in infants exposed to treated gestational diabetes mellitus (IGDM) and infants exposed to normal glucose tolerance (INGT) excluding the effect by the study used White's classification for diagnosis of gestational diabetes.

Author Contributions: Study protocol: M.P.H., K.D.K.A., J.M.B., A.P.H. and N.M.B.; Literature search: M.P.H., K.D.K.A. and J.M.B.; data extraction: M.P.H.; data verification: K.D.K.A. and J.M.B.; Quality assessment: M.P.H. and J.M.B.; meta-analysis: M.P.H.; preparation of the first draft of the manuscript: M.P.H.; reviewing and editing the manuscript: M.P.H., K.D.K.A., J.M.B., A.P.H. and N.M.B.; All authors have read and agreed to the published version of the manuscript.

Funding: This research received no external funding.

Institutional Review Board Statement: Not applicable.

Informed Consent Statement: Not applicable.

Data Availability Statement: Data sharing not applicable.

Acknowledgments: The authors are grateful to Ian Bollard, Research Services Librarian, University of Tasmania, for assistance in searching databases, Andrew Williams, School of Health Sciences, University of Tasmania for advice regarding the meta-analysis, and the authors listed in Table 1 who kindly provided additional data. This research was supported by an Australian Government Research Training Program (RTP) Scholarship to M.P.H.

Conflicts of Interest: The authors declare no conflict of interest.

\section{References}

1. Gyasi-Antwi, P.; Walker, L.; Moody, C.; Okyere, S.; Salt, K.; Anang, L.; Eduful, E.; Laryea, D.; Ottie-Boakye, D.; Asah-Opoku, K. Global Prevalence of Gestational Diabetes Mellitus: A Systematic Review and Meta-Analysis. New Am. J. Med. 2020, 1, 1-10.

2. Catalano, P.M. 10 Short and long term effects of gestational obesity: Clinical observations. Perinat. Program. 2011, 97-106. [CrossRef]

3. Nijs, H.; Benhalima, K. Gestational Diabetes Mellitus and the Long-Term Risk for Glucose Intolerance and Overweight in the Offspring: A Narrative Review. J. Clin. Med. 2020, 9, 599. [CrossRef]

4. Isganaitis, E. Developmental Programming of Body Composition: Update on Evidence and Mechanisms. Curr. Diabetes Rep. 2019, 19, 60. [CrossRef] 
5. Pedersen, J.; Osler, M. Hyperglycemia as the cause of characteristic features of the foetus and newborn of diabetic mothers. Dan. Med. Bull. 1961, 8, 78-83.

6. Desoye, G.; Nolan, C.J. The fetal glucose steal: An underappreciated phenomenon in diabetic pregnancy. Diabetology 2016, 59, 1089-1094. [CrossRef] [PubMed]

7. Catalano, P.M.; Thomas, A.; Huston-Presley, L.; Amini, S.B. Increased fetal adiposity: A very sensitive marker of abnormal in utero development. Am. J. Obstet. Gynecol. 2003, 189, 1698-1704. [CrossRef]

8. American Diabetes Association Diagnosis and Classification of Diabetes Mellitus. Diabetes Care 2013, 37, S81-S90. [CrossRef]

9. American Diabetes Association 14. Management of Diabetes in Pregnancy: Standards of Medical Care in Diabetes-2019. Diabetes Care 2018, 42, S165-S172. [CrossRef]

10. American Diabetes Association 2. Classification and diagnosis of diabetes: Standards of medical care in diabetes-2020. Diabetes Care 2020, 43, S14-S31. [CrossRef] [PubMed]

11. The HAPO Study Cooperative Research Group Hyperglycemia and Adverse Pregnancy Outcome (HAPO) Study: Associations with Neonatal Anthropometrics. Diabetes 2008, 58, 453-459. [CrossRef]

12. Chi, C.; Loy, S.L.; Chan, S.-Y.; Choong, C.; Cai, S.; Soh, S.E.; Tan, K.H.; Yap, F.; Gluckman, P.D.; Godfrey, K.M.; et al. Impact of adopting the 2013 World Health Organization criteria for diagnosis of gestational diabetes in a multi-ethnic Asian cohort: A prospective study. BMC Pregnancy Childbirth 2018, 18, 69. [CrossRef]

13. Daneshmand, S.S.; Stortz, S.; Morrisey, R.; Faksh, A. Bridging Gaps and Understanding Disparities in Gestational Diabetes Mellitus to Improve Perinatal Outcomes. Diabetes Spectr. 2019, 32, 317-323. [CrossRef]

14. American Diabetes Association 13. Management of Diabetes in Pregnancy:Standards of Medical Care in Diabetes-2018. Diabetes Care 2017, 41, S137-S143. [CrossRef]

15. Hartling, L.; Dryden, D.M.; Guthrie, A.; Muise, M.; Vandermeer, B.; Donovan, L. Benefits and Harms of Treating Gestational Diabetes Mellitus: A Systematic Review and Meta-analysis for the U.S. Preventive Services Task Force and the National Institutes of Health Office of Medical Applications of Research. Ann. Intern. Med. 2013, 159, 123-129. [CrossRef]

16. Yamamoto, J.M.; Kellett, J.E.; Balsells, M.; García-Patterson, A.; Hadar, E.; Solà, I.; Gich, I.; Van Der Beek, E.M.; CastañedaGutiérrez, E.; Heinonen, S.; et al. Gestational Diabetes Mellitus and Diet: A Systematic Review and Meta-analysis of Randomized Controlled Trials Examining the Impact of Modified Dietary Interventions on Maternal Glucose Control and Neonatal Birth Weight. Diabetes Care 2018, 41, 1346-1361. [CrossRef] [PubMed]

17. García-Patterson, A.; Balsells, M.; Yamamoto, J.M.; Kellett, J.E.; Solà, I.; Gich, I.; Van Der Beek, E.M.; Hadar, E.; CastañedaGutiérrez, E.; Heinonen, S.; et al. Usual dietary treatment of gestational diabetes mellitus assessed after control diet in randomized controlled trials: Subanalysis of a systematic review and meta-analysis. Acta Diabetol. 2018, 56, 237-240. [CrossRef]

18. Poolsup, N.; Suksomboon, N.; Amin, M. Effect of Treatment of Gestational Diabetes Mellitus: A Systematic Review and Meta-Analysis. PLoS ONE 2014, 9, e92485. [CrossRef]

19. Aisa, M.C.; Cappuccini, B.; Barbati, A.; Clerici, G.; Torlone, E.; Gerli, S.; Di Renzo, G.C. Renal Consequences of Gestational Diabetes Mellitus in Term Neonates: A Multidisciplinary Approach to the DOHaD Perspective in the Prevention and Early Recognition of Neonates of GDM Mothers at Risk of Hypertension and Chronic Renal Diseases in Later Life. J. Clin. Med. 2019, 8, 429. [CrossRef]

20. Åman, J.; Hansson, U.; Östlund, I.; Wall, K.; Persson, B. Increased Fat Mass and Cardiac Septal Hypertrophy in Newborn Infants of Mothers with Well-Controlled Diabetes during Pregnancy. Neonatology 2011, 100, 147-154. [CrossRef] [PubMed]

21. Lingwood, B.E.; Henry, A.M.; D’Emden, M.C.; Fullerton, A.-M.; Mortimer, R.H.; Colditz, P.B.; Le Cao, K.-A.; Callaway, L.K. Determinants of Body Fat in Infants of Women with Gestational Diabetes Mellitus Differ with Fetal Sex. Diabetes Care 2011, 34, 2581-2585. [CrossRef] [PubMed]

22. Au, C.P.; Raynes-Greenow, C.H.; Turner, R.M.; Carberry, A.E.; Jeffery, H.E. Body Composition Is Normal in Term Infants Born to Mothers with Well-Controlled Gestational Diabetes Mellitus. Diabetes Care 2012, 36, 562-564. [CrossRef]

23. Moher, D.; Liberati, A.; Tetzlaff, J.; Altman, D.G.; The PRISMA Group. Preferred reporting items for systematic reviews and meta-analyses: The PRISMA statement. PLoS Med. 2009, 6, e1000097. [CrossRef]

24. Covidence Systematic Review Software, Veritas Health Innovation, Melbourne, Australia. Available online: www.covidence.org (accessed on 25 March 2020).

25. Kennedy, C.E.; Fonner, V.A.; Armstrong, K.A.; Denison, J.A.; Yeh, P.T.; O’Reilly, K.R.; Sweat, M.D. The Evidence Project risk of bias tool: Assessing study rigor for both randomized and non-randomized intervention studies. Syst. Rev. 2019, 8, 1-10. [CrossRef]

26. Review Manager (RevMan) [Computer Program]. Version 5.4, The Cochrane Collaboration; The Nordic Cochrane Centre: Copenhagen, Denmark, 2020.

27. Enzi, G.; Inelmen, E.M.; Caretta, F.; Villani, F.; Zanardo, V.; DeBiasi, F. Development of Adipose Tissue in Newborns of Gestationaldiabetic and Insulin-dependent Diabetic Mothers. Diabetes 1980, 29, 100-104. [CrossRef]

28. Stevenson, D.K.; Ochikubo, C.G.; Rodgers, P.A.; Kerner, J.A., Jr. Anthropometry and bilirubin production. J. Perinatol. 1991, 11, 340-342.

29. Vohr, B.R.; McGarvey, S.T.; Coll, C.G. Effects of Maternal Gestational Diabetes and Adiposity on Neonatal Adiposity and Blood Pressure. Diabetes Care 1995, 18, 467-475. [CrossRef]

30. Simmons, D.; Robertson, S. Influence of maternal insulin treatment on the infants of women with gestational diabetes. Diabet. Med. 1997, 14, 762-765. [CrossRef] 
31. Okereke, N.C.; Uvena-Celebrezze, J.; Hutson-Presley, L.; Amini, S.B.; Catalano, P.M. The effect of gender and gestational diabetes mellitus on cord leptin concentration. Am. J. Obstet. Gynecol. 2002, 187, 798-803. [CrossRef]

32. Ng, P.C.; Lee, C.H.; Lam, C.W.K.; Wong, E.; Chan, I.H.S.; Fok, T.F. Plasma Ghrelin and Resistin Concentrations Are Suppressed in Infants of Insulin-Dependent Diabetic Mothers. J. Clin. Endocrinol. Metab. 2004, 89, 5563-5568. [CrossRef]

33. Westgate, J.A.; Lindsay, R.S.; Beattie, J.; Pattison, N.S.; Gamble, G.; Mildenhall, L.F.; Breier, B.H.; Johnstone, F.D. Hyperinsulinemia in Cord Blood in Mothers with Type 2 Diabetes and Gestational Diabetes Mellitus in New Zealand. Diabetes Care 2006, 29, 1345-1350. [CrossRef]

34. Rowan, J.A.; Hague, W.M.; Gao, W.; Battin, M.R.; Moore, M.P.; Mi, G.T.I. Metformin versus Insulin for the Treatment of Gestational Diabetes. N. Engl. J. Med. 2008, 358, 2003-2015. [CrossRef]

35. Lain, K.Y.; Garabedian, M.J.; Daftary, A.; Jeyabalan, A. Neonatal adiposity following maternal treatment of gestational diabetes with glyburide compared with insulin. Am. J. Obstet. Gynecol. 2009, 200, 501.e1-501.e6. [CrossRef] [PubMed]

36. Landon, M.B.; Spong, C.Y.; Thom, E.; Carpenter, M.W.; Ramin, S.M.; Casey, B.; Wapner, R.J.; Varner, M.W.; Rouse, D.J.; Thorp, J.M.; et al. A Multicenter, Randomized Trial of Treatment for Mild Gestational Diabetes. N. Engl. J. Med. 2009, 361, 1339-1348 [CrossRef] [PubMed]

37. Schaefer-Graf, U.M.; Meitzner, K.; Ortega-Senovilla, H.; Graf, K.; Vetter, K.; Abou-Dakn, M.; Herrera, E. Differences in the implications of maternal lipids on fetal metabolism and growth between gestational diabetes mellitus and control pregnancies. Diabet. Med. 2011, 28, 1053-1059. [CrossRef] [PubMed]

38. Buhling, K.J.; Doll, I.; Siebert, G.; Catalano, P.M. Relationship between sonographically estimated fetal subcutaneous adipose tissue measurements and neonatal skinfold measurements. Ultrasound Obstet. Gynecol. 2012, 39, 558-562. [CrossRef] [PubMed]

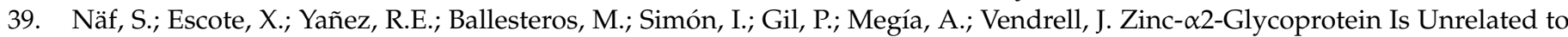
Gestational Diabetes: Anthropometric and Metabolic Determinants in Pregnant Women and Their Offspring. PLoS ONE 2012, 7, e47601. [CrossRef] [PubMed]

40. Brumbaugh, D.E.; Tearse, P.; Cree-Green, M.; Fenton, L.Z.; Brown, M.; Scherzinger, A.; Reynolds, R.; Alston, M.; Hoffman, C.; Pan, Z.; et al. Intrahepatic Fat Is Increased in the Neonatal Offspring of Obese Women with Gestational Diabetes. J. Pediatr. 2013, 162, 930-936.e1. [CrossRef]

41. Uebel, K.; Pusch, K.; Gedrich, K.; Schneider, K.-T.M.; Hauner, H.; Bader, B.L. Effect of maternal obesity with and without gestational diabetes on offspring subcutaneous and preperitoneal adipose tissue development from birth up to year-1. BMC Pregnancy Childbirth 2014, 14, 138. [CrossRef]

42. Kara, M.; Orbak, Z.; Döneray, H.; Ozkan, B.; Akcay, F. The Relationship Between Skinfold Thickness and Leptin, Ghrelin, Adiponectin, and Resistin Levels in Infants of Diabetic Mothers. Fetal Pediatr. Pathol. 2016, 36, 1-7. [CrossRef] [PubMed]

43. Mitanchez, D.; Jacqueminet, S.; Nizard, J.; Tanguy, M.-L.; Ciangura, C.; Lacorte, J.-M.; De Carne, C.; L'Hélias, L.F.; ChavattePalmer, P.; Charles, M.-A.; et al. Effect of maternal obesity on birthweight and neonatal fat mass: A prospective clinical trial. PLoS ONE 2017, 12, e0181307. [CrossRef]

44. Andersson-Hall, U.K.; Järvinen, E.A.J.; Bosaeus, M.H.; Gustavsson, C.E.; Hårsmar, E.J.; Niklasson, C.A.; Albertsson-Wikland, K.G.; Holmäng, A.B. Maternal obesity and gestational diabetes mellitus affect body composition through infancy: The PONCH study. Pediatr. Res. 2018, 85, 369-377. [CrossRef] [PubMed]

45. Maple-Brown, L.; Lee, I.-L.; Longmore, D.; Barzi, F.; Connors, C.; Boyle, J.A.; Moore, E.; Whitbread, C.; Kirkwood, M.; Graham, S.; et al. Pregnancy and Neonatal Diabetes Outcomes in Remote Australia: The PANDORA study—An observational birth cohort. Int. J. Epidemiol. 2018, 48, 307-318. [CrossRef]

46. Prentice, P.M.; Olga, L.; Petry, C.J.; Simmons, D.; Murphy, H.R.; Hughes, I.A.; Acerini, C.L.; Ong, K.K.; Dunger, D.B. Reduced size at birth and persisting reductions in adiposity in recent, compared with earlier, cohorts of infants born to mothers with gestational diabetes mellitus. Diabetologia 2019, 62, 1977-1987. [CrossRef]

47. Samsuddin, S.; Arumugam, P.A.; Amin, S.M.; Yahya, A.; Musa, N.; Lim, L.; Paramasivam, S.S.; Ratnasingam, J.; Ibrahim, L.; Chooi, K.C.; et al. Maternal lipids are associated with newborn adiposity, independent of GDM status, obesity and insulin resistance: A prospective observational cohort study. BJOG Int. J. Obstet. Gynaecol. 2019, 127, 490-499. [CrossRef] [PubMed]

48. Dauncey, M.J.; Gandy, G.; Gairdner, D. Assessment of total body fat in infancy from skinfold thickness measurements. Arch. Dis. Child. 1977, 52, 223-227. [CrossRef] [PubMed]

49. Weststrate, J.A.; Deurenberg, P. Body composition in children: Proposal for a method for calculating body fat percentage from total body density or skinfold-thickness measurements. Am. J. Clin. Nutr. 1989, 50, 1104-1115. [CrossRef]

50. Catalano, P.M.; Thomas, A.J.; Avallone, D.A.; Amini, S.B. Anthropometric estimation of neonatal body composition. Am. J. Obstet. Gynecol. 1995, 173, 1176-1181. [CrossRef]

51. Higgins, J.P.T.; Thompson, S.G.; Deeks, J.J.; Altman, D.G. Measuring inconsistency in meta-analyses. BMJ 2003, 327, 557-560. [CrossRef] [PubMed]

52. Coetzee, E.J. Pregnancy and diabetes scenario around the world: Africa. Int. J. Gynecol. Obstet. 2009, 104, S39-S41. [CrossRef]

53. Ryu, R.J.; Hays, K.E.; Hebert, M.F. Gestational diabetes mellitus management with oral hypoglycemic agents. Semin. Perinatol. 2014, 38, 508-515. [CrossRef]

54. Balsells, M.; García-Patterson, A.; Solà, I.; Roqué, M.; Gich, I.; Corcoy, R. Glibenclamide, metformin, and insulin for the treatment of gestational diabetes: A systematic review and meta-analysis. BMJ 2015, 350, h102. [CrossRef] 
55. Hiden, U.; Maier, A.; Bilban, M.; Ghaffari-Tabrizi, N.; Wadsack, C.; Lang, I.; Dohr, G.; Desoye, G. Insulin control of placental gene expression shifts from mother to foetus over the course of pregnancy. Diabetologia 2005, 49, 123-131. [CrossRef]

56. Hebert, M.F.; X Obstetric-Fetal Pharmacology Research Unit Network; Naraharisetti, S.B.; Krudys, K.M.; Umans, J.G.; Hankins, G.D.V.; Caritis, S.N.; Miodovnik, M.; Mattison, D.R.; Unadkat, J.D.; et al. Are We Optimizing Gestational Diabetes Treatment with Glyburide? The Pharmacologic Basis for Better Clinical Practice. Clin. Pharmacol. Ther. 2009, 85, 607-614. [CrossRef]

57. Nguyen, L.; Chan, S.-Y.; Teo, A.K.K. Metformin from mother to unborn child-Are there unwarranted effects? EBioMedicine 2018, 35, 394-404. [CrossRef]

58. Tarry-Adkins, J.L.; Aiken, C.E.; Ozanne, S.E. Comparative impact of pharmacological treatments for gestational diabetes on neonatal anthropometry independent of maternal glycaemic control: A systematic review and meta-analysis. PLoS Med. 2020, 17, e1003126. [CrossRef]

59. Tarry-Adkins, J.L.; Aiken, C.E.; Ozanne, S.E. Neonatal, infant, and childhood growth following metformin versus insulin treatment for gestational diabetes: A systematic review and meta-analysis. PLoS Med. 2019, 16, e1002848. [CrossRef] [PubMed]

60. Kwon, H.; Kim, D.; Kim, J.S. Body Fat Distribution and the Risk of Incident Metabolic Syndrome: A Longitudinal Cohort Study. Sci. Rep. 2017, 7, 1-8. [CrossRef]

61. Brumbaugh, D.E.; Friedman, J.E. Developmental origins of nonalcoholic fatty liver disease. Pediatr. Res. 2014, 75, 140-147. [CrossRef] [PubMed]

62. Gridneva, Z.; Hepworth, A.R.; Ward, L.C.; Lai, C.T.; Hartmann, P.E.; Geddes, D.T. Determinants of body composition in breastfed infants using bioimpedance spectroscopy and ultrasound skinfolds-Methods comparison. Pediatr. Res. 2016, 81, 423-433. [CrossRef] [PubMed]

63. Logan, K.M.; Emsley, R.J.; Jeffries, S.; Andrzejewska, I.; Hyde, M.J.; Gale, C.; Chappell, K.; Mandalia, S.; Santhakumaran, S.; Parkinson, J.R.; et al. Development of Early Adiposity in Infants of Mothers with Gestational Diabetes Mellitus. Diabetes Care 2016, 39, 1045-1051. [CrossRef] [PubMed]

64. Logan, K.M.; Gale, C.; Hyde, M.J.; Santhakumaran, S.; Modi, N. Diabetes in pregnancy and infant adiposity: Systematic review and meta-analysis. Arch. Dis. Child. Fetal Neonatal Ed. 2016, 102, F65-F72. [CrossRef] [PubMed] 